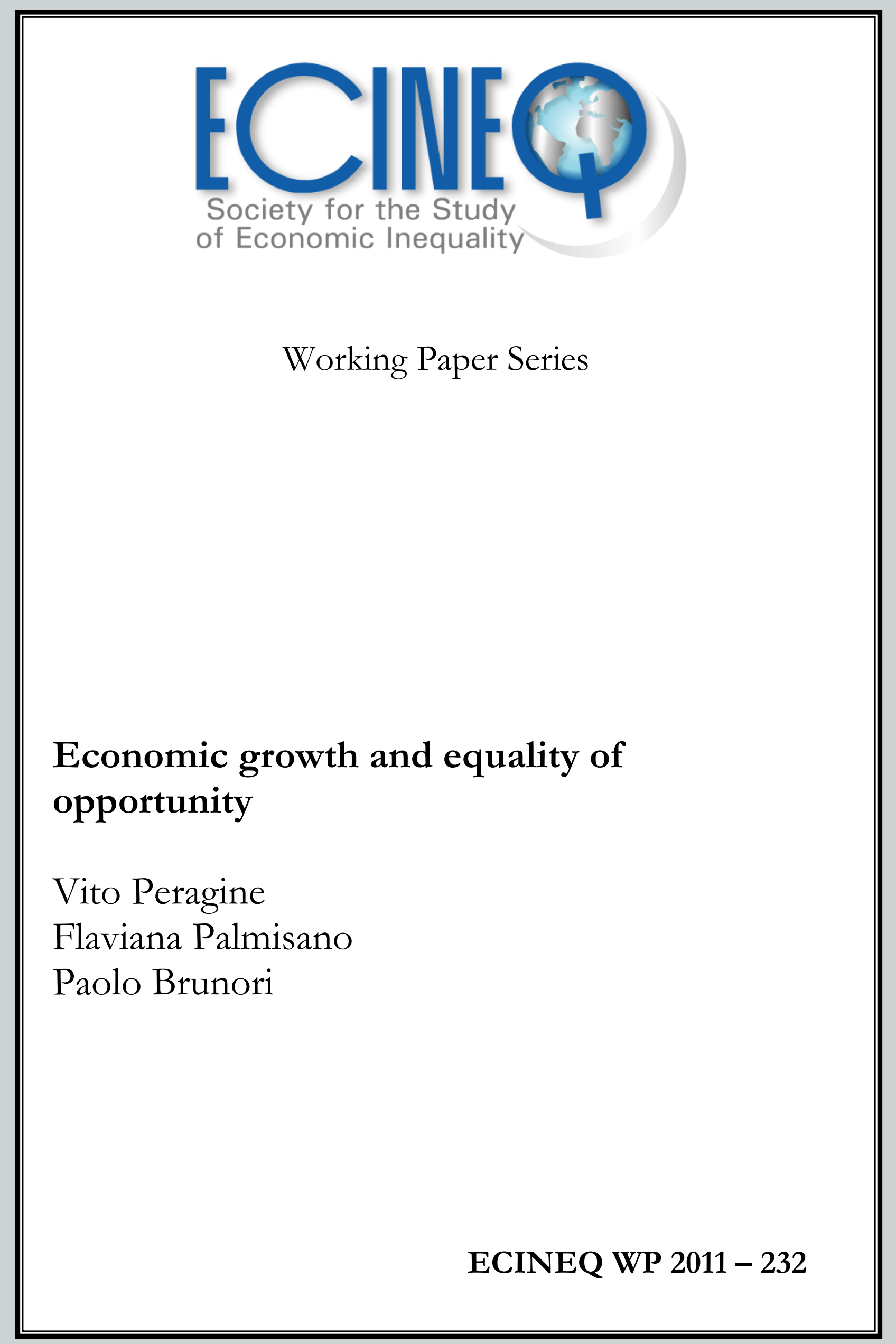




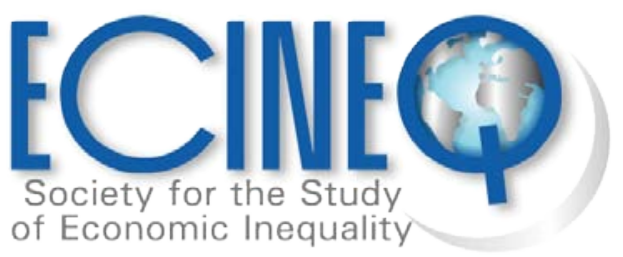

ECINEQ 2011 - 232
November 2011

www.ecineq.org

\title{
Economic growth and equality of opportunity $^{*}$
}

\author{
Vito Peragine ${ }^{\dagger}$ \\ Flaviana Palmisano \\ Paolo Brunori \\ Department of Economics and Mathematics, University of Bari
}

\begin{abstract}
In this paper we argue that a better understanding of the relationship between inequality and growth can be obtained by shifting the analysis from the space of final achievements to the space of opportunities. To this end, we introduce the Opportunity Growth Incidence Curve (OGIC) that can be used to evaluate the income dynamics of specific groups of the population and to infer the role of growth in the evolution of inequality of opportunity over time. By employing a rank dependent social welfare function we provide a rigorous normative justification for the use of the OGIC: We obtain suitable dominance conditions and we show how they can be interpreted in terms of OGIC dominance. We also propose an index that can be used to measure the impact of growth in terms of opportunity redistribution. Finally, we provide two empirical illustrations, for Italy and for Brazil, which show the potentials of the tools we have introduced.
\end{abstract}

Keywords: income inequality; inequality of opportunity; economic growth.

JEL classiffcation: D63, E24, O15, O40.

\footnotetext{
* Paper presented at the World Bank, OECD, 2011 abcde Conference "Broadening Opportunities for Development", held in Paris, May 30-June 1, 2011.

t Contact details: Vito Peragine, Department of Economics and Mathematics, University of Bari. v.peragine@dse.uniba.it.
} 


\section{Introduction}

In recent years, a central topic in the economic development literature is the measurement of the distributive impact of growth (see Bourguignon, 2003, 2004; Ferreira, 2010; Son, 2004; Ravallion and Chen, 2003). This literature has provided analytical tools in order to identify and quantify the effect of growth on distributional phenomena such as income (or consumption) poverty and income inequality: different indices for measuring the pro-poorness of growth have been proposed (see Grosse et al., 2008; Kakwani and Son, 2008; Klasen and Misselhorn, 2008; Kraay, 2006; Kakwani and Pernia, 2000; Essama-Nssah and Lambert, 2009) and the Growth Incidence Curve (GIC), measuring the quantile-specific rate of economic growth in a given period of time (Ravallion and Chen, 2003; Son 2004), has become a standard tool in evaluating growth 
from a distributional viewpoint. The interplay between growth, inequality and poverty reduction has also been investigated (Bourguignon, 2003; 2004).

All these tools are now used extensively in the field of development economics in order to evaluate and compare different growth processes in terms of social desirability and social welfare (see Atkinson and Brandolini, 2010; Datt and Ravallion, 2011; Lombardo, 2008; Araar et al., 2009).

A common feature of this literature is the focus on individual achievements as the proper "space" of distributional assessment: the distributional effects of growth are evaluated by looking at individual variables such as (equivalent) income or consumption.

On the other hand, a recent literature in the fields of normative economics has argued that equity judgements should be based on opportunities rather than observed outcomes (see Dworkin, 1981a,b; Sen, 1985; Cohen, 1989; Arneson, 1989; Roemer, 1998; Fleurbaey, 2008). The equal-opportunity framework stresses the link between the opportunities available to an agent and the initial conditions which are inherited or beyond the control of this agent. Proponents of equality of opportunity accept inequality of outcomes that arise from individual choices and effort, but do not accept inequalities of outcomes caused by circumstances beyond the individual control. This literature has motivated a rapidly growing number of empirical applications interested in measuring the degree of inequality of opportunity in a distribution and in evaluating public policies in terms of EOp (see, among others, Aaberge et al. 2011, Bourguignon et al., 2007; Checchi and Peragine 2010, Lefranc et al. 2009, Roemer et al. 2003). Book-length collections of empirical analysis of equality of opportunity in developing countries can be found in World Bank (2006) and de Barros et al. (2009).

The growing interest for equality of opportunity, in addition to the intrinsic normative justifications, is also motivated by instrumental reasons: it has been convincingly argued (see World Bank 2005 among others) that the degree of opportunity inequality in an economy could be related to the potentials for future growth. The idea is that when exogenous circumstances such as gender, race or parental background play a strong role in determining the individual income and occupation prospects, there is a suboptimal allocation of resources and lower potential for growth.

A better understanding of the relationship between inequality and growth, it is argued, can be obtainend by shifting the analysis from the space of final achievements to the space of opportunities.

The relationship between growth and inequality of opportunity is at center stage of our paper ${ }^{1}$ : we propose a framework and a set of simple tools that can be used to track the evolution of inequality of opportunity over time and to evaluate growth processes according to their impact on the degree of equality of opportunity. These tools can be used as complements to the standard analysis

\footnotetext{
${ }^{1}$ We investigate the relationship between growth and inequality of opportunity by a "micro approach"; an alternative "macro approach" would also be possible by investigating the relationship between growth and inequality of opportunity in a cross country or longitudinal perspective (see Marrero and Rodriguez, 2011).
} 
of the pro-poorness of growth and may give interesting insights for the design of public policies. If two growth process have, say, the same impact in terms of poverty and inequality reduction, but in the first case all members of a certain ethnic minority - or all people whose parents are illiterate - experience the lowest growth rate, while in another the poverty reduction is uncorrelated with differences in race or family background, our current arsenal of measures does not readily allow us to distinguish them.

To this end, we introduce the concept of Opportunity Growth Incidence Curve (OGIC). The OGIC plots the rate of income growth in a given period of time for each sub-group of the population, where the sub-groups are defined in terms of initial exogenous circumstances and are ordered from the poorest to the richest. We show the relationship between the OGIC and the degree of opportunity inequality: in general, a downward sloping OGIC indicates that growth contributes to equalizing opportunities over time, whereas an upward sloping curve indicates a growth that increases opportunity inequality. Then we propose a social welfare evaluation of different growth processes: using a rank dependent social welfare function expressing aversion to inequality of opportunity, we obtain suitable dominance conditions that can be used to rank growth processes in terms of their opportunity equalizing effect. We explore the relationship between these conditions and the OGIC and cumulative OGIC; we then propose a summary measure of the degree of opportunity redistribution induced by growth.

We finally provide an empirical illustration. The empirical implementation of the approach introduced is demanding in terms of data: in particular, it requires (i) a panel structure of income data and (ii) a rich set of information on the individual characteristics, including information on the individual background. As this rich set of information is often not available, especially for developing countries, we propose two empirical illustrations: our first analysis is based on the panel component of the Bank of Italy "Survey on Household Income and Wealth" (SHIW), which satisfies both requirements; the second empirical analysis is based on cross section data from Brazilian "Pesquisa Nacional por Amostra de Domicílios" (PNAD). The latter application is useful in order to show how to adapt our framework when a panel structure is not available.

The paper is organized as follows. Sections 2 and 3 briefly summarize the models and the tools used respectively in the literature on the distributional effect of growth and the inequality of opportunity literature; these constitute the basis of the subsequent analysis. Section 4 introduces and explains the properties of the OGIC. Section 5 contains the welfare analysis: it presents the dominance conditions and discusses the relationship with the OGIC. Section 5 also introduces a scalar index of opportunity inequality reduction. The empirical illustrations are contained in section 6 . Section 7 concludes. 


\section{The Growth Incidence Curve}

Let $\mathbf{y}_{t}$ be the distribution of income in a given population at time $t$, with density function $f\left(y_{t}\right)$ and cumulative distribution function (cdf) $F\left(y_{t}\right)$. Growth takes place over some time period, from $t$ to $t+1$. Let $y_{t}(p)$ be the quantile function of $F\left(y_{t}\right)$, representing the income level of the individuals ranked $p$ in $F\left(y_{t}\right)$, and let $y_{t+1}(p)$ be the quantile function of $F\left(y_{t+1}\right)$.

In order to evaluate growth and its distributional impact Ravallion and Chen (2003) define the Growth Incidence Curve (GIC) as follows:

$$
g(p)=\frac{y_{t+1}(p)}{y_{t}(p)}-1, \text { for all } p \in[0,1]
$$

The GIC plots the percentile specific rate of income growth against each percentile of the distribution, between two points in time. Clearly, $g(p) \geq 0$ $(g(p)<0)$ indicates a positive (negative) growth at $p$. Thus, it is possible to infer how growth impacted on each single point of the distribution, beyond simply looking at the overall mean income growth. The shape of the GIC, instead, provides information about the change in the level of inequality due to growth: a downward sloping GIC indicates that growth contributes to equalize the distribution of income (i.e. $g(p)$ decreases as $p$ increases), whereas an upward sloping GIC indicates a non-equalizing growth (i.e. $g(p)$ increases as $p$ increases). When the GIC is an horizontal line, inequality does not change over time and the rate of growth experienced by each quantile is equal to the rate of growth in the overall mean income, $\gamma=\frac{\mu\left(y_{t+1}\right)}{\mu\left(y_{t}\right)}-1$.

A cumulative representation of the GIC is provided by Son (2004) and it is defined as:

$$
G(p)=\frac{\int_{0}^{p} g(p) y_{t}(p)}{\int_{0}^{p} y_{t}(p)}, \text { for all } p \in[0,1]
$$

The cumulative GIC measures the change in mean income up to the $p$ poorest percentiles in the distribution. Thus, while GIC gives the rate of economic growth at each point of the distribution, the cumulative GIC plots the average income rate of growth up to the $p$ percentile. The $G(p)$ can be negatively or positively sloped depending on the fact that individuals may experience negative or positive growth. At $p=1, G(p)$ is equal to the change in the overall mean income.

To conclude this section, we notice that a specific aspect of the GIC has been recently criticized as unable to capture the welfare effect of growth: the GIC in fact compares the income levels of individuals in the same positions at time $t$ and $t+1$; but these could be (and typically will be) different individuals. Therefore the $G I C$ is unable to track the (welfare relevant) individual movements from 
period $t$ to period $t+1$. To capture this relevant effect, Grimm (2007) has ${ }^{2}$ introduced the non-anonymous GIC, defined by:

$$
n a-g\left(p_{t}\right)=\frac{y_{t+1}\left(p_{t}\right)}{y_{t}\left(p_{t}\right)}-1, \text { for all } p_{t} \in[0,1]
$$

where clearly $y_{t+1}\left(p_{t}\right)$ is the income at time $t+1$ of individuals ranked $p$ at time $t$. The non-anonymous ${ }^{3}$ GIC plots the rate of income growth against each percentile of the initial distribution, where the income refers to the same individuals in $t$ and $t+1$.

\section{The EOp model}

According to the EOp model, the individual income at a given time $t \in\{1, \ldots, T\}$, denoted by $y_{t}$, is a function of two sets of characteristics: the circumstances, $\mathbf{c}$, belonging to a finite set $\Omega=\left\{\mathbf{c}_{1}, \ldots, \mathbf{c}_{n}\right\}$, and the level of effort, $e_{t} \in \Theta \subseteq \Re_{+}$. The individual cannot be held responsible for $\mathbf{c}$, which is fixed over time; he is, instead, responsible for the effort $e_{t}$ he autonomously decides to exert in every period of time. Income is generated by a production function $g: \Omega \times \Theta \rightarrow \mathbb{R}_{+}$:

$$
y_{t}=g\left(c, e_{t}\right), \forall t \in\{1, \ldots, T\}
$$

This model excludes the existence of random components ${ }^{4}$. The function $g$ is assumed to be monotonic in circumstances and effort and it is the same for the whole population. The overall income distribution at time $t$ is $F\left(y_{t}\right)$.

We now introduce a partition of this distribution which is defined by the circumstances: each group in this partition is called a 'type' and includes all individuals sharing the same circumstances. Hence, for all $i=1, \ldots, n$, type $i$ is the set of individuals with circumstances $\mathbf{c}_{i}$; the outcome distribution of type $i$ at time $t$ is represented by a cdf $F_{i}\left(y_{t}\right)$, with population share $q_{i t}$ and mean $\mu_{i}\left(y_{t}\right)$. Hence, for all $t, F\left(y_{t}\right)=\sum_{i=1}^{n} q_{i t} F_{i}\left(y_{t}\right)$.

Given this analytical framework, the existing literature has proposed different definitions of equality of opportunity and, consequently, different approaches to measuring opportunity inequality. More specifically, the ex ante and the ex post approaches have been proposed in the literature (see van de Gaer 1993, Roemer, 1998, Fleurbaey and Peragine 2011). The ex post approach first identifies individuals at the same level of effort and than focuses on the outcome inequality among them. Any income inequality among individuals at the same effort level, for all effort levels, is interpreted as inequality of opportunity.

On the other hand, the ex ante approach focuses on the income prospects of individuals in the same type and interprets the type specific income distribution $F_{i}\left(y_{t}\right)$ as the set of opportunities open to individuals in type $i$. Consequently,

\footnotetext{
${ }^{2}$ See also Van Kerm (2009).

${ }^{3}$ See Bourguignon (2010), Jenkins and Van Kerm (2011) and Palmisano and Peragine (2011) for welfare-based justifications of the use of the non-anonymous GIC.

${ }^{4}$ See Lefranc et al. (2009) for a different model in which there is an additional factor, luck.
} 
according to the ex ante approach, there is equality of opportunity in a distribution $F\left(y_{t}\right)$ if the type specific distributions $\left(F_{1}, \ldots, F_{n}\right)$ have the same value; and the degree of opportunity inequality is evaluated by looking at the inequality between the type specific distributions.

A specific, utilitarian, version of the ex ante approach, which is extensively used in the applied literature and that will be used in this paper, further assumes that the value of the opportunity set $F_{i}\left(y_{t}\right)$ can be represented by the mean $\mu_{i}\left(y_{t}\right)$. Hence, given the original distribution $F\left(y_{t}\right)$, one focuses on the type mean distribution $F_{\mu}\left(y_{t}\right)=\left(\mu_{1}\left(y_{t}\right), \ldots, \mu_{n}\left(y_{t}\right)\right)$ : the degree of opportunity inequality in a distribution $F\left(y_{t}\right)$ is measured as the inequality in the distribution $F_{\mu}\left(y_{t}\right)$. Peragine (2002) and Aaberge et al. (2011) characterize on normative grounds the opportunity Lorenz curve associated to distribution $F_{\mu}\left(y_{t}\right)$, which can be defined as follows:

$$
L_{\mu t}\left(\frac{i}{n}\right)=\frac{\sum_{j=1}^{i} q_{j t} \mu_{j}\left(y_{t}\right)}{\sum_{j=1}^{n} q_{j t} \mu_{j}\left(y_{t}\right)}, \forall i=1, \ldots, n
$$

$L_{\mu t}$ captures the extent of the inequality between types; it can be used to rank snapshot income distributions according to inequality of opportunity. Different scalar measures of inequality of opportunity have also been introduced in the literature (see Aaberge et al. 2001, Checchi and Peragine 2010, Ferreira and Gignoux 2011): they are generally based on the inequality in the type mean distribution $F_{\mu}\left(y_{t}\right)$.

\section{The Opportunity GIC}

In this section we combine the theoretical tools introduced in sections 2 and 3 and introduce the concept of Opportunity Growth Incidence Curve. The OGIC can be considered as an alternative to the GIC for assessing the distributional features of growth, when the statistical units are represented by the types and an opportunity egalitarian perspective is adopted. The analysis tries to capture how growth affects differently the outcome prospects of individuals with different circumstances. The ex ante utilitarian approach implies that our framework will depend on the mean income of each type.

In analogy with the standard GIC, an Opportunity Growth Incidence Curve should plot the rate of income growth of each type, starting from the poorest type, up to the richest type.

Now, while the composition of the types does not change from one period to another (as we are assuming that the circumstances are fixed over time), it might well be the case that, after the growth process, the ranking of the types in terms of their average income does change. Should we compare the average income of the types that occupy the same position in the two periods of time, 
hence adopting an anonymous version of the OGIC? Or, rather, should we plot the growth in mean income of each type, where the types are sorted according to their position in the first period?

As our aim is to evaluate the evolution of the opportunities of individuals over time, we require that types, in both the pre and the post growth distributions, be sorted according to their mean income in the initial distribution, independently of their position in the final distribution. Hence the following analysis assumes non-anonymity of types.

Let $F_{\mu}\left(y_{t}\right)$ be the distribution of type mean income at time $t$ with $\mu_{1}\left(y_{t}\right) \leq$ $\ldots \leq \mu_{n}\left(y_{t}\right)$ and $\tilde{F}_{\mu}\left(y_{t}\right)=\left(\tilde{\mu}_{1}\left(y_{t+1}\right), \ldots, \tilde{\mu}_{n}\left(y_{t+1}\right)\right)$ the distribution of type mean income at time $t+1$ where types are ordered according to their position at time $t$.

The Opportunity Growth Incidence Curve is defined by:

$$
g_{i}^{o}=\frac{q_{i t+1} \tilde{\mu}_{i}\left(y_{t+1}\right)-q_{i t} \mu_{i}\left(y_{t}\right)}{q_{i t} \mu_{i}\left(y_{t}\right)}, \forall i=1, \ldots, n
$$

The OGIC plots the type specific mean income growth rate against each type $i=1, \ldots, n$, keeping the ranking of the types constant over time. Clearly $g_{i}^{o}$ may be positive or negative depending on whether types experience income gains or $\operatorname{losses}^{5}$. When $g_{i}^{o} \geq 0\left(g_{i}^{o}<0\right)$ for each $i=1, \ldots, n$, that is, when each type mean increases (decreases), everyone faces better income prospects after growth: a situation of pareto-improvement (worsening).

Note that if $q_{i t+1}=q_{i t}$, as it should be in the case of non-anonymity, then simply $g_{i}^{o}=\frac{\tilde{\mu}_{i}\left(y_{t+1}\right)-\mu_{i}\left(y_{t}\right)}{\mu_{i}\left(y_{t}\right)}$.

The shape of the OGIC allows to assess how growth differs between social groups. It is horizontal if growth is proportional. It is negatively sloped if growth is progressive, that is, it favours more the initially poorer types than those initially richer. When the OGIC is positively sloped growth is regressive, since it favors initially richer to initially poorer types.

The relationship between the OGIC and the Opportunity Lorenz Curve is given by:

$$
g_{i}^{o}=\frac{\Delta_{i} \tilde{L}_{\mu t+1}\left(\frac{i}{n}\right)}{\Delta_{i} L_{\mu t}\left(\frac{i}{n}\right)}(\gamma+1)-1
$$

Where $\tilde{L}_{\mu t+1}\left(\frac{i}{n}\right)$ is the concentration curve of the post growth type mean income and $\Delta_{i} L_{\mu t}\left(\frac{i}{n}\right)=\frac{q_{i t} \mu_{i}\left(y_{t}\right)}{\mu\left(y_{t}\right)}$ is the first derivative with respect to $i$ of the Opportunity Lorenz Curve defined in eq. (4).

Through eq. (6) it is possible to infer how growth affects the level of inequality of opportunity. When growth does not have any impact on the level of inequality of opportunity, there is no change in the first derivative of the Lorenz

\footnotetext{
${ }^{5}$ Note that, since we adopt a proportionate growth rate, the growth rate in each type-mean income is different from the average of individual income growth rates. Similarly, the average of the type mean income growth is different from the overall mean income growth when we adopt a proportionate OGIC. Instead, the overall mean income growth is equal to the average of the type mean income growth weighted by their initial mean income.
} 
curve, hence the OGIC equals the overall mean income growth for each type. By contrast, when growth causes a variation in the level of opportunity inequality, hence a change in the first derivative of the Lorenz curve, the OGIC will differ from the overall mean income growth rate. In particular, $g_{i}^{o}>\gamma\left(g_{i}^{o}<\gamma\right)$ as long as the income share of the type, i.e. $\frac{q_{i t} \mu_{i}\left(y_{t}\right)}{\mu\left(y_{t}\right)}$, increases (decreases) over time $^{6}$.

We also propose a cumulative version of the OGIC, obtained as the proportionate growth rate of the partial mean of the types. The cumulative OGIC can be defined by:

$$
G_{i}^{o}=\frac{\sum_{j=1}^{i} g_{j}^{o} q_{j t} \mu_{j}\left(y_{t}\right)}{\sum_{j=1}^{i} q_{j t} \mu_{j}\left(y_{t}\right)}
$$

The cumulative OGIC plots the mean income growth rate up to type $i$ against each cumulated type in the population. It can be downward or upward sloping, depending on the pattern of growth among types. Clearly, at $i=n, G_{i}^{o}$ equals the overall mean income growth rate, $\gamma$.

Combining eq. (8) with eq. (4) gives the following result:

$$
G_{i}^{o}=\left(\frac{\tilde{L}_{\mu t+1}\left(\frac{i}{n}\right)}{L_{\mu t}\left(\frac{i}{n}\right)}(\gamma+1)\right)-1
$$

The decomposition in eq. (9) allows to express the cumulative OGIC as depending on two components: the overall mean income change and the variation in the level of the inequality of opportunity. In case of proportional growth, the Lorenz curve and the concentration curve do not change and the cumulative OGIC is equal to overall mean income growth rate. In general, the overall mean income growth rate is given by a weighted average of each type income growth weighted by their initial mean income.

\section{A social welfare evaluation of growth}

The aim of this section is to develop dominance conditions to rank growth processes from the EOp perspective and to provide a normative justification for

\footnotetext{
${ }^{6}$ In the above analysis we have assumed non-anonymity. When one instead moves to keep the anonymity assumtion then the OGIC is defined by:

$$
g_{i}^{o}=\frac{\Delta_{i} L_{t+1}\left(\frac{i}{n}\right)}{\Delta_{i} L_{t}\left(\frac{i}{n}\right)}(\gamma+1)-1
$$

Note that, instead of using the concentration curve for period $t+1$, in the equation above we use the Lorenz curve since now types are ordered according to their level of final income. In this case a variation in the inequality of opportunity can be due not only to a change in the relative distance between a given type mean income and the overall mean income, but also to a variation of a type specific population share.
} 
the use of the OGIC.

Let $x=y_{t+1}-y_{t}$ be the individual income variation between $t$ and $t+1$. Since in our model circumstances are fixed over time, individuals belonging to a given type $i$ at time $t$ will be the same individuals belonging to type $i$ at time $t+1$. Therefore we denote by $F_{i}(x)$ the cdf of the income change of individuals of type $i$. Moreover, let $x_{i}(p)$ be the quantile function of $F_{i}(x)$, representing the income change of the individual ranked $p$ in $F_{i}(x)$, with $\mu_{i}(x)$ denoting the mean income variation (in levels) of individuals belonging to type $i$. Hence, $\mu_{i}(x)$ represents the extent of growth in the value of the opportunity set of the individuals of type $i$. Let the growth process $F\left(y_{t} \mid c\right) \longrightarrow F\left(y_{t+1} \mid c\right)$ be denoted by $F(x \mid c)$; it describes the income transformation process of all individuals conditional on their type. Let the set of all admissible growth processes be denoted by $D$.

We adopt a rank-dependent approach to the normative evaluation of growth ${ }^{7}$ (Yaari, 1988). According to this formulation, the social evaluation of growth of individuals belonging to the same type $i$ is represented by a weighted sum of ordered income changes, where each income change is weighted according to its position in the ranking, that is:

$$
W\left(F_{i}(x)\right)=\int_{0}^{1} v(p) x_{i}(p) d p, \forall i=1, \ldots, n
$$

The function $v(p):[0,1] \longrightarrow \Re_{+}$expresses the social weight of any income change ranked $p$ in $F_{i}(x)$.

In order to evaluate the overall growth process we aggregate the social welfare evaluation of growth experienced by each type weighted by the relevant population share, using type-specific weighting functions. We obtain the following social welfare function (SWF):

$$
W(F(x \mid c))=\sum_{i=1}^{n} q_{i} \int_{0}^{1} v_{i}(p) x_{i}(p) d p
$$

The set of weight functions $\left\langle v_{1}(p), \ldots, v_{n}(p)>\right.$ will be called a weight profile and will be denoted by $V$. Different value judgments coherent with the EOp theory are expressed in this framework by imposing some properties on the "social weight" functions, hence selecting different classes of weight profiles. These in turn define different classes of social welfare functions.

Let us start with a standard monotonicity assumption.

Axiom 1 (Monotonicity) For all $i=1, \ldots, n$, for all $p \in[0,1]$

$$
v_{i}(p) \geq 0
$$

This axiom states that positive growth does not reduce social welfare, while a negative growth does not increase social welfare. Let $V_{\mathbf{1}}=\left\{\left\langle v_{1}(p), \ldots, v_{m}(p)\right\rangle\right.$ :

\footnotetext{
${ }^{7}$ See Aaaberge (2000) for a normative justification of the rank dependent approach to inequality analysis and Peragine (2002), Aaberge et al. (2011) and Palmisano (2011) for an application in the field of inequality of opportunity.
} 
Axioms 1 holds $\}$ and let $\mathrm{W}_{1}$ be the class of SWFs constructed as in (11) and based on weights profiles in $V_{1}$.

The following property expresses the social irrelevance of the difference in the income variation due to effort, that in our model is captured by the variation within the same type.

Axiom 2 (Effort neutrality) For all $p \in[0,1]$, for all $i=1, \ldots, n, \exists \beta_{i} \in$ $\Re_{+}$such that

$$
v_{i}(p)=\beta_{i}
$$

Imposing axiom 2 makes the weight only depend on the type, as required by the ex ante approach. A social planner endorsing this kind of preference, would be neutral with respect to differences in the level of growth experienced by individuals with the same circumstances.

Let $V_{\mathbf{1 2}}=\left\{<v_{1}(p), \ldots, v_{m}(p)\right\rangle$ : Axioms 1 and 2 hold $\}$ and let $\mathrm{W}_{12}$ be the class of SWFs constructed as in (11) and based on weights profiles in $V_{12}$.

The following property expresses the social relevance of progressive growth.

Axiom 3 (Opportunity inequality aversion) For all $p \in[0,1]$, for all $i=1, \ldots, n-1$

$$
v_{i}(p) \geq v_{i+1}(p)
$$

According to axiom 3 , social welfare does not decrease if an individual ranked $p$ in $F_{i}(x)$ experiences a higher growth than the individual ranked $p$ in $F_{i+1}(x)$. This axioms expresses aversion toward inequality between types. It states that social welfare is increased more the poorer is the type within which an individual experiences a positive growth. Let $V_{\mathbf{1 2 3}}=\left\{\left\langle v_{1}(p), \ldots, v_{m}(p)\right\rangle\right.$ : Axioms 1,2 and 3 hold $\}$ and let $\mathrm{W}_{123}$ be the class of SWFs constructed as in (11) and based on weights profiles in $V_{123}$.

We now turn to identify a range of conditions to be satisfied for ensuring growth dominance in terms of social welfare, for sets of SWFs satisfying the properties introduced above. We begin by imposing axioms 1 and 2 .

Proposition 1 For all growth process $F_{A}(x \mid c), F_{B}(x \mid c) \in D, W_{A} \geq$ $W_{B}, \forall W \in \mathbf{W}_{12}$ if and only if

$$
q_{A i} \mu_{A i}(x) \geq q_{B i} \mu_{B i}(x), \forall i=1, \ldots, n
$$

According to proposition 1 we need to verify that the mean income change of individuals in a given type $i$, weighted by the respective population share, is higher for $A$ than for $B$, and this must be checked for every type.

To see the relationship between the social welfare dominance in Proposition 1 and the opportunity growth incidence curve notice that, $\forall i=1, \ldots, n$,

$$
q_{A i} \mu_{A i}(x) \geq q_{B i} \mu_{B i}(x) \Leftrightarrow q_{A i} \mu_{A i}\left(y_{t}\right) g_{A i}^{o} \geq q_{B i} \mu_{B i}\left(y_{t}\right) g_{B i}^{o}
$$

The condition in eq. (13) is a first order dominance to be applied on the OGIC weighted by the initial level of income. An implication of this condition is that if the comparison concerns growth processes taking place on the same base-year distribution, the dominance condition in proposition 1 would be equivalent to the simple OGIC dominance. Hence, the result in proposition 1 
gives normative justification to the use of the OGIC in ranking growth processes taking place on the same initial distribution.

We now turn to the class of social welfare functions satisfying axiom 1, 2 and 3 .

Proposition 2 For all growth processes $F_{A}(x \mid c), F_{B}(x \mid c) \in D, W_{A} \geq$ $W_{B}, \forall W \in W_{123}$ if and only if

$$
\sum_{i=1}^{k} q_{A i} \mu_{A i}(x) \geq \sum_{i=1}^{k} q_{B i} \mu_{B i}(x), \forall k=1, \ldots, n
$$

According to proposition 2 we need to verify that the cumulated mean income change of individuals in a given type $i$, weighted by the respective population share, is higher for $A$ than for $B$, and this must be checked at every step.

The dominance condition expressed in Proposition 2 is equivalent to:

$$
\sum_{i=1}^{k} q_{A i} g_{A i}^{o} \mu_{A i}\left(y_{t}\right) \geq \sum_{i=1}^{k} q_{B i} g_{B i}^{o} \mu_{B i}\left(y_{t}\right), \forall k=1, \ldots, n
$$

Thus we have to check that the cumulative OGIC weighted by the initial level of income for the growth process $A$ must lay nowhere below the cumulative absolute OGIC for the growth process $B$, and this dominance must be checked at every step. Again, in case of growth processes taking place on the same initial distribution of income, the dominance condition in proposition 2 would be equivalent to the dominance of the respective cumulative OGIC.

\subsection{An index of opportunity equalizing growth}

The dominance conditions established above provide robust but only partial rankings of growth processes. Complete rankings, instead, can be obtained adopting scalar measures and this is the aim of this section.

First, by considering the social evaluation function endorsing a social preference for opportunity equalizing growth, i.e. $W \in \mathbf{W}_{123}$, we can write eq. (11) as follows:

$$
W^{*}(F(x \mid c))=\sum_{i=1}^{n} q_{i} v_{i} \mu_{i}(x)
$$

The weight function in eq. (16) satisfies the following properties: $\forall i=1, \ldots, n$, $v_{i} \geq 0$ and $v_{i} \geq v_{i+1}$. As a result, $W^{*}(F(x \mid c))$ can be used to measure the extent of growth, which is also sensitive to its progressivity (opportunity equalizing effect); that is, the higher the growth experienced by the initially poorer types with respect to those initially richer, the higher the social evaluation of the growth process.

Further observing that in case of proportional growth eq. (16) becomes: $\bar{W}(F(x \mid c))=\bar{\mu}(x) \sum_{i=1}^{n} q_{i} v_{i}$, we can use $\bar{W}(F(x \mid c))$ as a benchmark to evaluate the extent to which growth acts by equalizing opportunities. Thus, an 
index of opportunity equalizing growth can be defined by:

$$
O G=\frac{\sum_{i=1}^{n} q_{i} v_{i} \mu_{i}(x)}{|\bar{\mu}(x)| \sum_{i=1}^{n} q_{i} v_{i}}-1
$$

where $\bar{\mu}(x)=\sum_{i=1}^{n} q_{i} \mu_{i}(x)$ is the overall mean income growth and can be interpreted as the growth every type would experience in case of proportionality. $O G$ measures the relative distance between the effective process of growth and an hypothetical proportional process of growth.

Hence, $O G$ can be interpreted as a measure of the incidence of growth in alleviating (worsening) economic disparities among individuals of different types. A positive value of the index, $O G>0$, indicates a progressive process, that is growth is concentrated more among types ranked lower in the initial distribution of income. A negative value of the index, $O G<0$, indicates a regressive process, that is income growth is concentrated more among richer types. $O G=0$ indicates proportional growth.

Clearly the formulation above defines a class of indices which depend on the specific sets of weights $\left(v_{1}, \ldots, v_{n}\right)$ one chooses. A special member of this class is obtained by adopting the following parametrization: $v_{i}=1-\sum_{j=1}^{i} q_{j}$; in this case eq. (17) becomes a Gini-type measure of opportunity equalization:

$$
G=\frac{\sum_{i=1}^{n} q_{i}\left(1-\sum_{j=1}^{i} q_{j}\right) \mu_{i}(x)}{|\bar{\mu}(x)| \sum_{i=1}^{n} q_{i}\left(1-\sum_{j=1}^{i} q_{j}\right)}-1
$$

$G$ has the advantage of lying between minus one and one: $G=1$ when all the growth is concentrated on the poorest type, while $G=-1$ when growth is concentrated on the richest type.

We also propose an absolute version of the Gini-type measure of progressivity, obtained multiplying $O G$ by the average growth:

$$
a G=\frac{\sum_{i=1}^{n} q_{i}\left(1-\sum_{j=1}^{i} q_{j}\right) \mu_{i}(x)}{\sum_{i=1}^{n} q_{i}\left(1-\sum_{j=1}^{i} q_{j}\right)}-\bar{\mu}(x)
$$


This index is equal to 0 in case of proportional growth; it is positive in case of opportunity equalizing growth and negative in case of opportunity inequality increasing growth. In case of total regressivity, $a O G=-|\bar{\mu}(x)|$, in case of total progressivity: $a O G=+|\bar{\mu}(x)|$.

Thus, this index can be interpreted as the gain or loss in social welfare due to the progressivity or regressivity of growth. A positive value of the index can be interpreted as the gain in social welfare due to the equalization of opportunity over time, while a negative value is associated to a loss in social welfare.

\section{Two empirical exercises}

The empirical implementation of the approach presented in the previous sections is demanding in terms of data. Because of the longitudinal framework adopted, the social welfare evaluation of growth requires a panel structure of income data. Moreover, detailed information on the household characteristics are needed in order to partition the population into types. Our first empirical illustration is based on the panel component of the Bank of Italy "Survey on Household Income and Wealth" (SHIW), which satisfies both requirements. In the next section we propose an empirical illustration for the case in which a panel structure is not available - which is a frequent case for developing countries.

\subsection{Opportunity and growth in Italy}

The SHIW is a representative sample of the Italian resident population interviewed every two years. The unit of observation is the household, defined as all persons sharing the same dwelling, and the information reported in the 1998, 2002, and 2006 waves includes: family composition, number of siblings, the educational attainment, the occupational as well as the labour market activity status of respondents mother and father. The individual outcome is measured as household per capita disposable income. Income includes all household earnings, transfers, pensions, and capital incomes, net of taxes and social security contributions.

We consider eight years, from 1998 to 2006, and we divide the period in two time spells. The attempt is to evaluate the distributional impact of growth in terms of opportunities in 1998-2002 and in 2002-2006. The exercise is appealing as in the former time spell the government was held by a leftwing coalition while in the latter by a rightwing coalition ${ }^{8}$.

The panel components include 1,972 households in (1998-2002), 1,787 in (20022006), and 1,172 in (1998-2006); the average household per capita income growth

\footnotetext{
${ }^{8}$ Note that the panel components present in the 1998-2002, 2002-2006 and 1998-2006 waves are not exactly the same due to the survey design. A number of households exit from the sample and others are included. Moreover, the survey probability sample weights of each family varies over time introducing additional variability. We based our estimate on the probability weights as suggested in Faiella and Gambacorta (2007) in case of longitudinal analysis.
} 
in the three time periods was $4.79 \%, 2.68 \%$ and $6.36 \%$; complete descriptive statistics are reported in appendix.

The GICs for the two periods represent our benchmark figure ${ }^{9}$.

\section{FIGURE 1 ABOUT HERE}

The overall income growth rate is higher in the first period and the two growth processes show quite different patterns. Between 1998 and 2002 the income dynamic is moderately progressive (the GIC is decreasing), although growth soars for the richest quantile, while in the latter period growth is concentrated on the richer quantiles. The two patterns are symmetric, especially in the first half of the distribution, and cross around the median, favoring the households sitting at the intermediate quantile. However, the two growth processes cannot be unambiguously ordered because the confidence intervals for the GIC coordinates, reported in figure 9 in appendix, are quite large ${ }^{10}$.

\subsubsection{Opportunity Growth Incidence Curve}

The first step in the analysis of inequality of opportunity is the identification of types ${ }^{11}$. We base the partition in types on three characteristics of the head of the family ${ }^{12}$ : the highest education attainment (divided in two levels: up to elementary school, secondary or higher), the highest occupational status of her parents (not in the labour force and blue collar, white collar, executive) and the geographical area of birth (North, Centre, and South). Hence we have 18 types. Tables 1 and 2 in appendix report the full opportunity-profiles for 1998 and 2002 , the base-years of the two processes compared. The opportunity profile is a list of the types, where for each type there is a description of the specific circumstances defining it, the population share and the mean income (Ferreira and Gignoux). The types are ranked according to the average income. A look at the evolution of the opportunity profiles gives important information. The opportunities faced by individuals seem to be remarkably spatially concentrated. A majority of the individuals with lowest opportunity sets are natives of the South of Italy, and this happens both in 1998 (the poorest four types) and in 2002 (the poorest 3 types). Second, family background is also crucial in the identification of the smallest opportunity sets. Indeed, most of the lower-ranked

\footnotetext{
${ }^{9}$ The number of quantiles is 50 .

${ }^{10}$ The standard errors to compute the $95 \%$ confidence intervals of the curve are obtained through 1000 bootstrap replications. We adopt the algorithm programmed by Michael Lokshin (World Bank, 2011 see the section "Achieving Shared Growth - Growth Incidence Curve" at http : //web.worldbank.org).

${ }^{11}$ Data limitation prevent from exactly observing all circumstances that affect household income and impose a selection of a subset of the entire bunch of circumstances beyond individual control. This choice has the consequence that the inequality of opportunity estimates represent a lower bound of the real inequality of opportunity in the income distribution (see Ferreira and Gignoux, 2010 and Luongo, 2011 for a discussion of this issue).

12 The limited sample size of the SHIW panel component prevents us to define a finer partition.
} 
types comprise people whose parents have at least elementary education ${ }^{13}$. By contrast, the occupational status of the parents does not appear to be critical in the determination of the lower ranked types.

We also notice that some reranking of types takes place over time, especially in the the second process. For example, the type composed by individuals from the Centre, with parents working as executive but with a low educational attainments, bares the worst "downward" repositioning. By contrast, the type composed by individuals born in the North, whose parents belong to the lowest job category and attained higher educational levels, experiences a substantial "upward" repositioning.

Figure 2 shows the (non-anonymous) OGIC ${ }^{14}$. The 1998-2002 and 2002-2006 curves are reported with $95 \%$ confidence intervals in figure 10 in appendix.

\section{FIGURE 2 ABOUT HERE}

In the first period, with the exception of one type (the type ranked 4 in 1998), all types in the lower part of the distribution experience positive growth, while most of the types ranked higher face a negative - although modest change in mean income, with the exception of the richest type. Hence, growth appears to be more beneficial for types at the tails of the distribution in 1998; that is, for individuals born in south regions whose parents have low level of educational attainments and unqualified job, and for individuals from the north whose parents work as executive and attained higher educational levels. While people form the centre, with high educated parents working as executive or withe collar appears to be harmed by growth.

As concerned the second process, almost every type experiences an increase in the mean income ${ }^{15}$, but the richest type, that faces a decrease in the mean income. Types gaining more from growth are those individuals born in the centre, whose parents have at most the elementary education level but working as executive or withe collar, and also those born in the south but whose parents have higher educational level and works as executive. Whereas, types suffering from a decrease in income are more heterogeneous, they comprise individuals from the centre with low educated parents but working as executive, and individuals from the south with high educated parents but working as blue-collar or not in the labour force.

Looking at the overall process, it is not straightforward whether the growth dynamics are regressive or progressive in terms of opportunity; in fact, both OGICs are neither monotonically increasing nor decreasing (figure 2). As far as the ordering of growth processes is concerned, the OGIC dominance is based on the sign of the vertical distance between the two curves, for each type. As

\footnotetext{
${ }^{13}$ An exceptions is the poorest types in 1998 , but it accounts for a very little proportion of the population.

${ }^{14}$ The standard errors for the curve coordinates obtained by 1000 ordinary non parametric bootstrap replications (Davison et al., 1986). The confidence interval is: $\gamma_{i}^{O}=$ $\hat{g}_{i}^{O} \pm z_{1-\alpha / 2} \hat{S E}\left(\hat{g}_{i}^{O}\right)$, where $\hat{S E}$ is the standard error of the 1000 bootstrap replication of the statistic (Fox and Weisberg, 2002).

${ }^{15}$ Note that changes tend to be smaller in absolute value than in the first time period.
} 
shown in figure 10, this difference has opposite and statistically significant signs, that is the two OGICs cannot be ordered.

A final observation concerns the comparison with the GIC. The first process shows that there are specific groups of the population facing negative growth, while, looking at the standard GIC, growth appears to be always positive. This suggests that to adopt an EOp approach to evaluate growth may be a useful complement to the standard tools of analysis. A GIC with a progressive pattern may be associated to an OGIC with a regressive one. This does not represent a contradiction, on the contrary signals how the focus of the two tools differ: while for the GIC is inequality in income per se that matter while in the OGIC case is the composition of the inequality between types that is relevant.

We report the cumulative OGICs in figure 3.

\section{FIGURE 3 ABOUT HERE}

The overall mean income growth rates - that can be read as the coordinate of the richest type - are rather different, however the two patterns are similar. Both curves fall steeply up to the poorest types, take a slightly convex shape in very first part of the type means distribution and concave up to the 11 type, then, become flat up to richest type. Again, it is not immediate to grasp their redistributive effect in terms of opportunity. Nevertheless, if we exclude the poorest type, their comparison suggests a clear dominance of the first period over the second.

\subsubsection{Dominance test}

In this section we test the conditions proposed in proposition 1 and 2 . The two propositions are equivalent to a test of dominance of the OGIC and cumulative OGIC weighted by the initial level of income respectively; the two curves are represented in figures 4 and 5 . Both curves do not display a clear-cut shape and cross many times. The pattern of the curves in proposition 2 (figure 5 ) is more sharp-cut.

\section{FIGURE 4 AND 5 ABOUT HERE}

The first period shows a highly concave shape up to around the $60 \%$ of distribution, becoming convex after that. Whereas, the second period presents a number of inflection points along the whole distribution. This observation suggests that the first process is more effective in equalizing opportunity (in absolute terms).

The tests proposed in proposition 1 and 2 are sequential tests for difference in means. In the first case we test for the difference in the absolute change in per capita income weighted by the population share of each type, in the second for the difference in the partial means of change weighted by the population share of the first $i$ types.

In the first case we reject the null hypothesis of zero difference in means for the majority of types, however no dominance is found because the difference 
in means is statistically significant but has opposite sings for different types. The second test, instead, allows us to rank the two growth processes. The distance between the two curves turns out to be statistically significant in types 8 and 9; hence, we can state that the 1998-2002 growth process second order dominates the 2002-2006 growth process according to all opportunity equalizing social welfare function defined in eq. (12).

\subsubsection{Opportunity equalizing growth index}

In this section we obtain a complete ranking of the growth processes by adopting the $O G$ index defined in equation (18). The complete ranking clearly indicates that the 1998-2002 growth process has been more opportunity equalizing than the second time spell. Table 1 reports estimates for the absolute $\left(O G_{A B S}\right)$ and relative $\left(O G_{R E L}\right)$ version of the index and their difference.

\section{TABLE 1 ABOUT HERE}

The second order dominance of the first growth process over the second is confirmed by our estimates. The difference between the two indexes is positive, i.e. $O G$ is higher for the first period, and statistically significant. Moreover, estimate for the index is positive for the 1998-2002 period and is negative for the 2002-2006 indicating progressivity for the former process and regressivity for the latter.

\subsection{Opportunity and growth in Brazil}

Our theoretical framework may be of great interest in the analysis of developing and emerging economies that experience lively growth processes and whose income dynamics have dramatic impact on poverty and redistribution. Unfortunately such empirical applications are severely restrained by lack of data. However, our approach may be interestingly implemented also using non longitudinal data. A number of waves of a cross section survey for example can be used to draw anonymous OGIC curves. Once the population is partitioned in types, repeated cross section information are sufficient to track the income dynamic of types. By contrast we cannot track the income dynamics of households, which implies that we cannot apply the entire set of criteria we have proposed in section 4 and 5, relying the latter on the assumption of non-anonymity.

To illustrate this point, in this section we use the yearly Brazilian Pesquisa Nacional por Amostra de Domicílios (PNAD) to estimate the shape of the GIC, OGIC and cumulative OGIC curves. The PNAD is a national representative household survey from which we select the waves 2002, 2005 and 2008. The cross section datasets include 109,864 households in 2002, 121,014 in 2005 and 118,890 in 2008. Income is defined as monthly per capita household income, expressed in current Brazilian real. Household income are computed as the sum of all household members' individual incomes, including earning from all jobs, and all other reported incomes, including those from assets, pensions and transfers. 
We divide the six year period in two three year periods: 2002-2005 and 20052008. In this case the choice is driven by the diverging economic trend during the two time spells. The former was a period of economic slow down for Brazil. In particular, PNAD data record a reduction of the average income between 2002 and 2003 and a per capita disposable income in 2005 only $2.08 \%$ higher than in 2002. The latter period is instead a period of pronounced growth with a mean growth ten times higher (20.2\% between 2005 and 2008).

As for the first empirical exercise, we start the analysis with the representation of the benchmark figure: the GIC for the two periods considered. Figure 6 reports the GICs based on 50 quantiles ${ }^{16}$.

\section{FIGURE 6 ABOUT HERE}

The overall mean income growth rate is higher in the second period, income change is generally positive with the exception of the richest quantiles who experience negative growth in the first period. The two growth processes show a very similar and clear pattern. Their trend is also very clear, both the income dynamics in 2002-2005 and 2005-2008 are progressive (the respective GICs are neatly decreasing), thus growth is concentrated more on the poorer quantiles. Furthermore, it is possible to order unambiguously the two growth processes, since the confidence intervals for the GIC coordinates, reported in figure 13 in the appendix, are quite small and never overlap ${ }^{17}$.

\subsubsection{Opportunity Growth Incidence Curve}

In this section we apply the anonymous version of the framework introduced in section 4. The first step is, again, the identification of types. Given the information available in the survey we only consider two circumstances: region of birth and race. The former is coded in 5 categories (North, Northeast, Southeast, South, Center-west) and the latter in three (white/east Asians, black/mixed race, indigenous). We exclude individuals who were born abroad and those classified as "other" in the variable race, as the number of observations was too low to make appropriate inferences. Individuals sharing the same region of birth and race form a type. We thus have 15 types.

Table 5 and 6 in appendix report the full opportunity-profiles for 2002 and 2005 , the base-years of the two processes compared, the population share of each type, its mean per capita income. Some types present large population shares, these are the poorest and the richest type for 2002 and types 2 (type 1 in 2002) and 15 for 2005, while others represent smaller part of the population. Moreover, the type population share $\left(q_{i}\right)$ varies over time, that is $q_{i}$ cannot be assumed fixed during the growth process.

It is clear that the race is the main determinant of the disparity in opportunities in 2002. Most of the lowest ranked types comprise households characterized

\footnotetext{
${ }^{16}$ For all curves we adopt the same estimation procedure used for the Italian case.

${ }^{17}$ The comparison with the Italian GICs makes clear the improvement in terms of sample size and hence in terms of confidence intervals for GICs. This may be considered among the reasons to choose a cross section dataset rather than a panel.
} 
by a black-mixed head. In particular the poorest type is represented by blackmixed individuals born in Northeast. This feature is less pronounced in 2005. The poorest type in 2005 is represented by indigenous born in Center west. The other poorer types comprise households from the North - Northeast area with black-mixed or indigenous head.

Focussing on the richest type, both in 2002 and 2005 it comprises white-east Asian individuals born in the Southeast. Looking at the rest of the upper part of the distribution, race is still determinant in shaping household opportunities, the higher mean income is hold by White-East Asian households; their geographical origin is instead more heterogeneous.

Figure 7 reports the OGIC for the two three-year periods. Because the population share varies across time we have drawn an unweighted version of the two OGICs.

\section{FIGURE 7 ABOUT HERE}

One feature stands out: the almost exact symmetry of the two curves. The interpretation is that types gaining more in one period are those gaining less in the other. With respect to the first period, growth appears to be more beneficial for the first two poorest types in 2002; that is, for households originated from Northeast regions, with indigenous or black-mixed head. While households with black-mixed head, coming from South or Center-west regions appear to be harmed by growth.

With respect to the second process, types gaining more from growth comprise households with indigenous head, born in North or South regions. Whereas, types gaining less are households with black-mixed head, coming form South or Southeast regions. However both OGICs show a progressive pattern in terms of opportunity.

The dominance of the 2005-2008 period is clear. The difference in the OGIC coordinates is statistically significant for almost all types as shown in figure 14 in appendix.

Figure 8 reports the cumulative OGIC.

\section{FIGURE 8 ABOUT HERE}

The two curves tend to be downward sloping confirming that the both growth processes are opportunity improving. As expected the comparison highlights a clear dominance of the second period over the first, this difference is statistically significant as shown in figure 15 in the appendix ${ }^{18}$.

A last observation concerns the comparison between the OGIC and the standard GIC. Although the two curves show the same progressive pattern, their behaviour over the entire distribution is different. Indeed, the GIC is smoothly decreasing, whereas the OGIC presents a number of peacks all along the distribution. This is mostly evident by looking at the second type. While adopting

\footnotetext{
${ }^{18}$ Note that in this case the cumulative OGIC is not weighted by the population share and hence the last coordinate cannot be interpreted as the average income growth in the period.
} 
the standard GIC there is a neat dominance of the second process over the first, this cannot be proved anymore when implementing the OGIC. In fact, the coordinates of the two OGICs for that type are almost the same, implying that the dominance of the growth rate of that type in the second period is not anymore statistically different from the growth experied by the same type in the first process.

\section{Conclusions}

In this paper we have argued that a better understanding of the relationship between inequality and growth can be obtainend shifting the analysis from final achievements to opportunities.

To this end, we have introduced the Opportunity Growth Incidence Curve, that can be used to evaluate the income dynamics of specific groups of the population and to infer the role of growth in the evolution of inequality of opportunity over time. We then have provided a normative justification for the use of the OGIC by employing a rank dependent and opportunity egalitarian social welfare function. Indeed, the welfare dominance conditions we have obtained can be interpreted in terms of initial income weighted OGIC dominance. Adopting a specific configuration of this social welfare function we have also proposed an index (both in a relative and absolute version) that can be used to measure the impact of growth in terms of opportunity redistribution.

In the second part of the study, we have provided two empirical illustrations, for Italy and for Brazil. These illustrations show the potentials of the tools we have introduced and show that these tools can be used also when, as it is often the case in developing countries, tha available data do not meet the strong requirement of the theoretical framework.

Another field of application of our framework is the analysis of tax-benefit reforms. Typically, the distributional aspects of these reforms are analyzed through 'microsimulation' techniques and are evaluated in terms of income inequality reduction. Comparing reforms with the help of the tools developed in this paper which allow evaluating the inequality of opportunity reduction seems a promising path for future research. 


\section{References}

[1] Aaberge, R. (2000). Characterizations of Lorenz Curves and Income Distributions, Social Choice and Welfare 17, 639-653.

[2] Aaberge, R., M. Mogstad, \& Peragine, V. (2011). Measuring Long-term Inequality of Opportunity, Journal of Public Economics, 95(3-4), 193-204.

[3] Araar, A., Duclos, J.-Y., Audet, M. and Makdissi, P. (2009). Testing for pro-poorness of growth, with an application to Mexico. Review of Income and Wealth, 55: 853-881.

[4] Arneson, R. (1989). Equality of Opportunity for Welfare. Philos. Stud, 56, $77-93$.

[5] Atkinson, A. B. and Brandolini, A. (2010). On Analyzing the World Distribution of Income, World Bank Econ Rev, 24(1).

[6] Barros, R., Ferreira, F., Vega, J. and Chanduvi, S. (2009). Measuring Inequality of Opportunity in Latin America and the Caribbean. Washington, DC: The World Bank.

[7] Bourguignon, F. (2003). The Growth Elasticity of Poverty Reduction: Explaining Heterogeneity across Countries and Time Periods. In: Eicher, T., Turnovsky, S. Inequality and growth: theory and policy implications. MIT Press, Cambridge.

[8] Bourguignon, F. (2004). The Poverty-Growth-Inequality Triangle. Indian Council for Research on International Economic Relations, Working Paper N.125.

[9] Bourguignon, F. (2010). Non-anonymous Growth Incidence Curves, Income Mobility and Social Welfare Dominance. Journal of Economic Inequality, DOI: $10.1007 / \mathrm{s} 10888-010-9159-7$.

[10] Bourguignon F. \& Ferreira F. H. G. \& Menéndez M., 2007. "Inequality Of Opportunity In Brazil," Review of Income and Wealth, Vol. 53(4), pages 585-618.

[11] Checchi, D., \& Peragine, V. (2010). Inequality of Opportunity in Italy. Journal of Economic Inequality, 8 (4), 429-450.

[12] Cohen, G.A. (1989). On the Currency of Egalitarian Justice. Ethics, 99, 906-944.

[13] Datt, G. and Ravallion, M. (2011). Has India's Economic Growth Become More Pro-Poor in the Wake of Economic Reforms? World Bank Econ Rev, $25(2)$.

[14] Davison, A.C., Hinkley, D.V. and Schechtman, E. (1986). Efficient bootstrap simulation. Biometrika, 73, 555 - 566. 
[15] Dworkin, R. (1981a). What is equality? Part 1: Equality of welfare. Part 2: Equality of resources, Philos. Public Affairs, 10, 185-246.

[16] Dworkin, R. (1981b). What is equality? Part 1: Equality of welfare. Part 2: Equality of resources, Philos. Public Affairs, 10, 283-345.

[17] Essama-Nssah, B., Lambert, P. (2009). Measuring Pro-poorness: a Unifying Approach with New Results, Review of Income and Wealth, 55, 3, 752-778.

[18] Faiella I., Gambacorta R. (2007). The weighting process in the SHIW, Banca d'Italia, Temi di discussione del Servizio Studi, Number 636 - June 2007.

[19] Ferreira, F. H. G. (2010). Distributions in motion. Economic growth, inequality, and poverty dynamics. World Bank, Policy research working paper N. 5424 .

[20] Ferreira, Francisco H. G. and Jérémie Gignoux, (2011). The Measurement of Inequality of Opportunity: Theory and an Application to Latin America, The Review of Income and Wealth (forthcoming).

[21] Fleurbaey, M. (2008). Fairness, Responsibility and Welfare, 1st Edition. Oxford University Press.

[22] Fleurbaey M. and Peragine V. (2011). Ex ante versus ex post equality of opportunity, Economica (forthcoming)

[23] Fox J., Weisberg S. (2002). An R and S-PLUS Companion to Applied Regression, Sage Publication, Thausand Oaks, California.

[24] Grimm, M. (2007). Removing the anonymity axiom in assessing pro-poor growth, Journal of Economic Inequality, 5(2), 179-197.

[25] Grosse, M., Harttgen, K., Klasen, S. (2008). Measuring Pro-poor Growth in Non-income Dimensions, World Development, 36, 6, 1021-1047.

[26] Jenkins, S., Van Kern, P. (2006). Trends in income inequality, pro-poor income growth and income mobility, Oxford Economic Papers, 58, 3, 53148.

[27] Jenkins, S., Van Kerm, P. (2011). Trends in individual income growth: measurement methods and British evidence. IZA DP. No. 5510.

[28] Kakwani, N., Pernia, E. (2000). What is Pro-poor Growth?, Asian Development Review, 18, 1-16.

[29] Kakwani, N., Son, H. (2008). Poverty Equivalent Growth Rate, Review of Income and Wealth, 54, 4, 643-655. 
[30] Klasen, S., Misselhorn, M. (2008). Determinants of the Growth Semielasticity of Poverty Reduction, EUDN, Working Paper N. 2008-11.

[31] Kraay, A. (2006). When is Growth Pro-poor? Evidence From a Panel of Countries, Journal of Development Economics, 80, 1, 198-227.

[32] Lefranc, A., Pistolesi, N., \& Trannoy, A. (2009). Equality of opportunity and luck: Definitions and testable conditions, with an application to income in France. Journal of Public Economics, 93(11-12), 1189-1207.

[33] Lombardo, V. (2008). Growth and Inequality effects on poverty, Journal of Income Distribution.

[34] Luongo, P. (2011). The implication of partial observability of circumstances on the measurement of IOp, Research on Economic Inequality, Volume 19 (forthcoming).

[35] Marrero G. A. and Rodríguez J. G. (2010). Inequality of opportunity and growth, Working Papers 154, ECINEQ, Society for the Study of Economic Inequality.

[36] Palmisano F. (2011). Mobility and long term equality of opportunity, Research on Economic Inequality, Volume 19 (forthcoming).

[37] Palmisano F., Peragine V. (2011). The distributional incidence of growth: a non-anonymous and rank dependent approach, University of Bari, mimeo.

[38] Peragine, V. (2002). Opportunity Egalitarianism and Income Inequality: the Rank-Dependent Approach, Mathematical Social Sciences, 44, 45-64.

[39] Peragine, V. (2004a). Ranking income distributions according to equality of opportunity, Journal of Economic Inequality, 2(1), 11-30.

[40] Peragine, V. (2004b). Measuring and Implementing Equality of Opportunity for Income, Social Choice and Welfare, 22, 1-24.

[41] Ravallion, M., Chen, S. (2003). Measuring pro-poor growth, Economics Letters, 78, 1, 93-9.

[42] Roemer, J. (1998). Equality of Opportunity. Cambridge, MA: Harvard University Press.

[43] Roemer, J.E., Aaberge, R., Colombino, U., Fritzell, J., Jenkins, S., Lefranc, A., et al. (2003a). To what extent do fiscal systems equalize opportunities for income acquisition among citizens?, Journal of Public Economics 87: $539-565$.

[44] Sen A. 1985, Commodities and Capabilities, North-Holland, Amsterdam.

[45] Son, H. H. (2004). A note on pro-poor growth, Economics Letters, 82, 3, 307-314. 
[46] Van de Gaer, D. (1993) Equality of opportunity and investment in human capital. Ph.D. Dissertation, Katholieke Universiteit Leuven.

[47] Van Kerm, P. (2009). Income mobility profiles, Economic Letters, 102, 2, 93-95.

[48] Yaari, M. (1988). A Controversial Proposal Concerning Inequality Measurement, Journal of Economic Theory, 44, 381-397.

[49] World Bank. (2005). World Development Report 2006: Equity and Development. Washington, DC: World Bank.

\section{Appendix}

Before proving this proposition we need to prove the following lemma.

Lemma $1 \sum_{k=1}^{n} v_{k} w_{k} \geq 0$ for all sets of numbers $\left\{v_{k}\right\}$ such that $v_{k} \geq v_{k+1} \geq 0$ $\forall k \in\{1, \ldots, n\}$ if and only if $\sum_{i=1}^{k} w_{i} \geq 0, \forall k \in\{1, \ldots, n\}$.

Proof. Applying Abel's decomposition: $\sum_{k=1}^{n} v_{k} w_{k}=\sum_{k=1}^{n}\left(v_{k}-v_{k+1}\right) \sum_{i=1}^{k} w_{i}$. If $\sum_{i=1}^{k} w_{i} \geq 0, \forall k \in\{1, \ldots, n\}$, then $\sum_{k=1}^{n} v_{k} w_{k} \geq 0$. As for the necessity part, suppose that $\sum_{k=1}^{n} v_{k} w_{k} \geq 0$ for all sets of numbers $\left\{v_{k}\right\}$ such that $v_{k} \geq v_{k+1} \geq 0$, but $\exists j \in\{1, \ldots, n\}: \sum_{i=1}^{j} w_{i}<0$. Consider what happens when $\left(v_{k}-v_{k+1}\right) \searrow$ $0, \forall k \neq j$. We obtain that $\sum_{k=1}^{n} v_{k} w_{k} \longrightarrow\left(v_{j}-v_{j+1}\right) \sum_{i=1}^{j} w_{i}<0$, a contradiction.

Proof of Proposition 1

We want to find a necessary and sufficient condition for

$$
\Delta W=\sum_{i=1}^{n}\left(q_{A i} \int_{0}^{1} v_{i}(p) x_{A i}(p)-q_{B i} \int_{0}^{1} v_{i}(p) x_{B i}(p) d p\right) \geq 0, \forall W \in \mathbf{W}_{12}
$$

For the sufficiency, by axiom $3 v_{i}(p)=\beta_{i}, \forall p \in[0,1]$ and $\forall i=1,2, \ldots, n$, therefore we can write eq. (19) as follows: 


$$
\begin{aligned}
\Delta W= & \sum_{i=1}^{n} \beta_{i}\left[q_{A i} \int_{0}^{1} x_{A i}(p) d p-q_{B i} \int_{0}^{1} x_{B i}(p) d p\right]= \\
= & \sum_{i=1}^{n} \beta_{i}\left[q_{A i} \mu_{A i}(x)-q_{B i} \mu_{B i}(x)\right] \geq 0
\end{aligned}
$$

by axiom $1 v_{i}(p)=\beta_{i} \geq 0, \forall p \in[0,1]$ and $\forall i=1,2, \ldots, n, \Delta W \geq 0$ if $q_{A i} \mu_{A i}(x)-q_{B i} \mu_{B i}(x) \geq 0, \forall i=1, \ldots, n$.

For the necessity, suppose that

$$
\Delta W=\sum_{i=1}^{n} \beta_{i}\left[q_{A i} \mu_{A i}(x)-q_{B i} \mu_{B i}(x)\right] \geq 0
$$

but $\exists k=1, \ldots, n$ such that $q_{A k} \mu_{A k}(x)<q_{B k} \mu_{B k}(x)$. We can choose a set of numbers $\left\{\beta_{i}\right\}_{i=1, \ldots, n}$ such that $\beta_{i} \searrow 0, \forall i \neq k . \Delta W$ would reduce to $\beta_{k}\left(q_{A k} \mu_{A k}(x)-q_{B k} \mu_{B k}(x)\right)<0$, a contradiction. QED

\section{Proof of Proposition 2}

We want to find a necessary and sufficient condition for

$$
\Delta W=\sum_{i=1}^{n}\left(q_{A i} \int_{0}^{1} v_{i}(p) x_{A i}(p)-q_{B i} \int_{0}^{1} v_{i}(p) x_{B i}(p) d p\right) \geq 0, \forall W \in \mathbf{W}_{123}
$$

For both conditions, note that by axiom 3 we can write: $\Delta W=\sum_{i=1}^{n} \beta_{i}\left[q_{A i} \mu_{A i}(x)-q_{B i} \mu_{B i}(x)\right] \geq$ 0 . Let $S_{i}=\left[q_{A i} \mu_{A i}(x)-q_{B i} \mu_{B i}(x)\right], \forall i=1, \ldots, n$. Since by axiom $2 \beta_{i} \geq \beta_{i+1}$ and $\beta_{i} \geq 0$ by axiom 1 , we can apply Lemma 1 . Therefore, $\Delta W=\sum_{i=1}^{n} \beta_{i} S_{i} \geq 0$ if and only if $\sum_{i=1}^{k} S_{i} \geq 0, \forall k=1, \ldots, n$. Hence, $\Delta W \geq 0$ if and only if $\sum_{i=1}^{k} q_{A i} \mu_{A i}(x)-q_{B i} \mu_{B i}(x), \forall k=1, \ldots, n$. QED 


\section{Figures and Tables (Text)}

Figure 1: Italian GICs 1998-2002-2006. Source: Authors' calculation from SHIW.

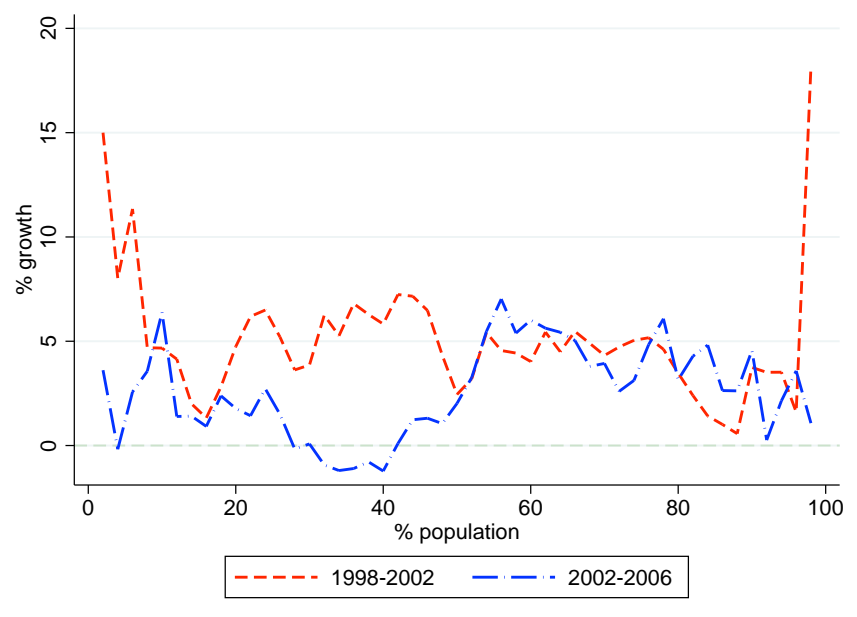

Figure 2: Italian OGICs 1998-2002-2006 (non-anonymous). Source: Authors' calculation from SHIW.

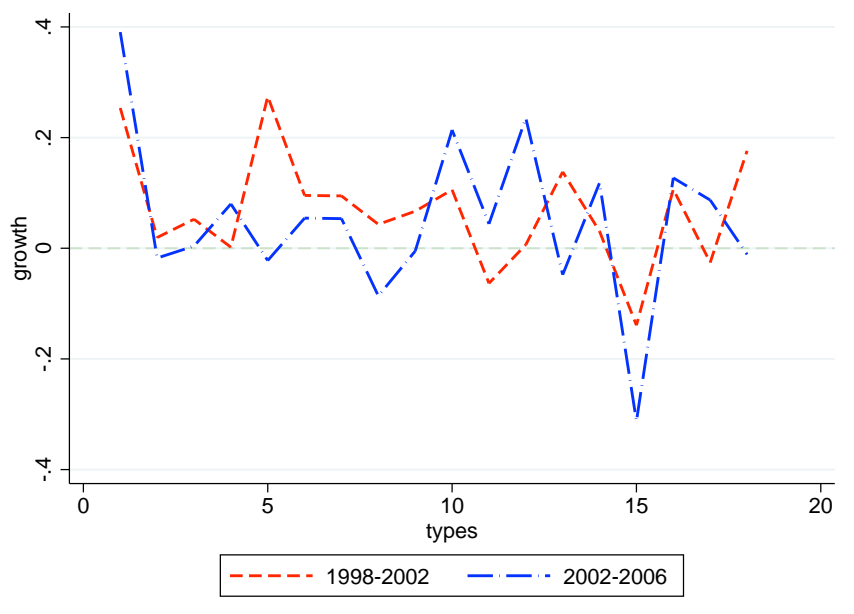


Figure 3: Italian cumulated OGICs 1998-2002-2006. Source: Authors' calculation from SHIW.

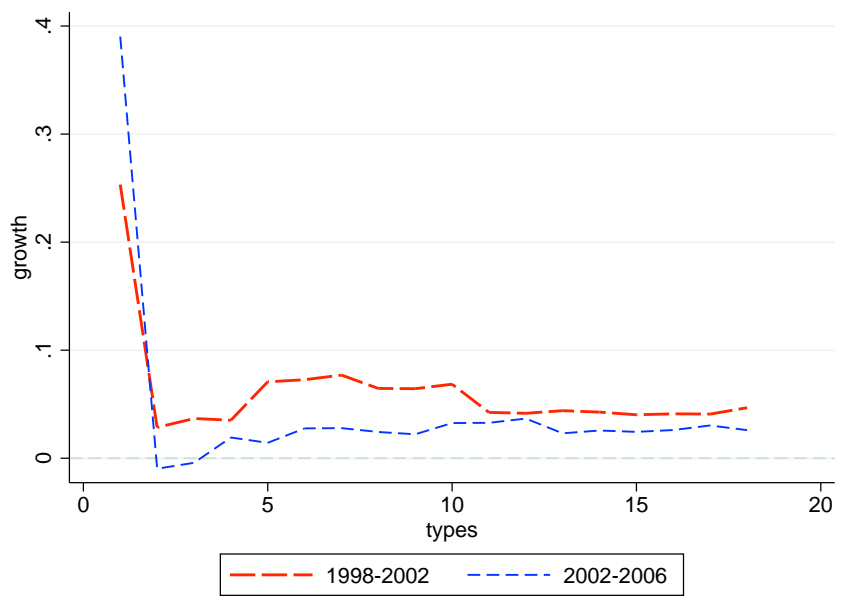

Figure 4: Proposition 1: OGICs 1998-2002-2006. Source: Authors' calculation from SHIW

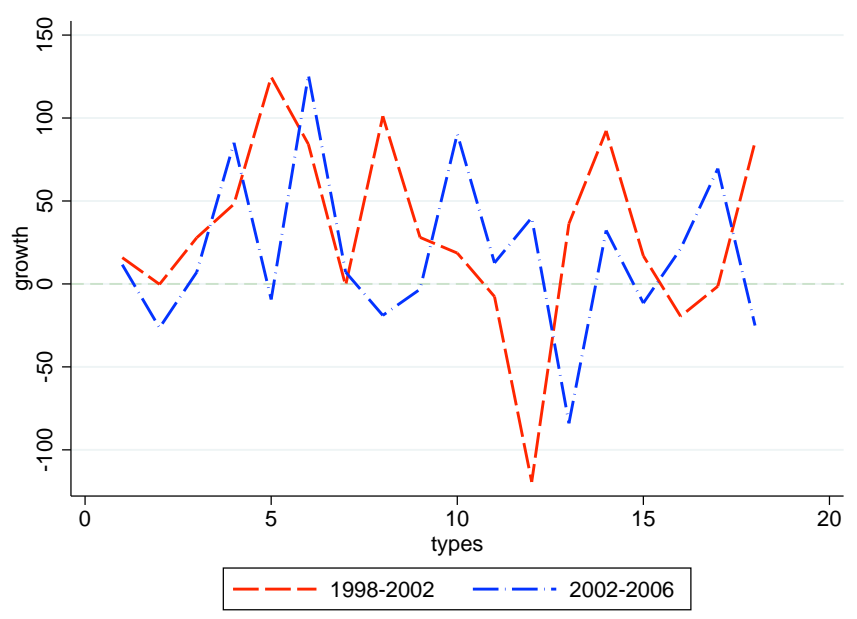


Figure 5: Proposition 2: OGICs 1998-2002-2006. Source: Authors' calculation from SHIW

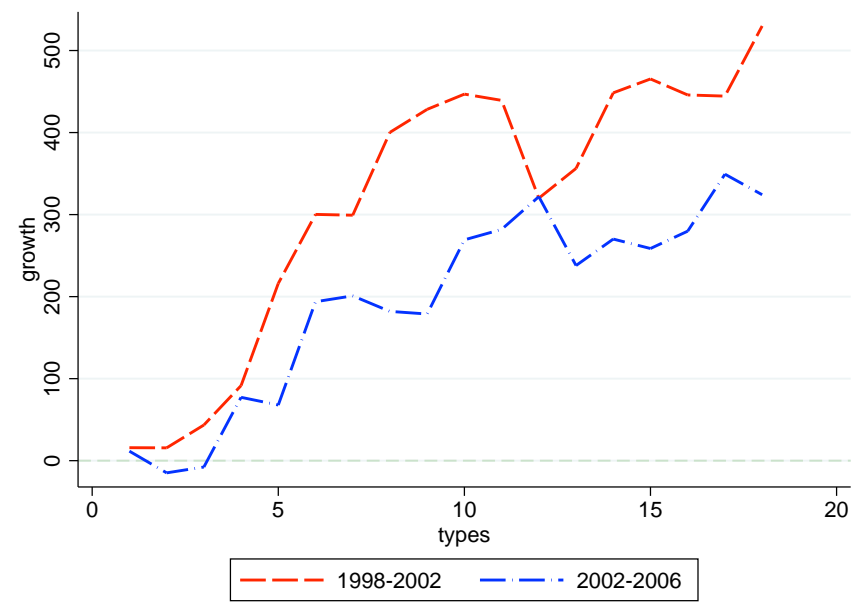

Table 1: $O G$ index with $95 \%$ confidence intervals.

\begin{tabular}{c|lll|lll}
\hline period & $O G_{R E L}^{L}$ & $\overline{O G_{R E L}}$ & $O G_{R E L}^{L}$ & $O G_{A B S}^{L}$ & $\overline{O G_{A B S}}$ & $O G_{A B S}^{L}$ \\
\hline \hline $1998-2002$ & 0.0826 & 0.1239 & 0.1651 & 52.622 & 59.63 & 66.64 \\
$2002-2006$ & -0.2111 & -0.1519 & -.0926 & -79.71 & -63.79 & -47.87 \\
$1998-2006$ & -0.0177 & -0.0126 & -0.0075 & -13.56 & -9.97 & -6.38 \\
\hline $98 / 02-02 / 06$ & 0.2041 & 0.2758 & 0.3475 & 106.25 & 123.42 & 140.61 \\
\hline
\end{tabular}


Figure 6: Brazilian GICs 2002-2005-2008. Source: Authors' calculation from PNAD.

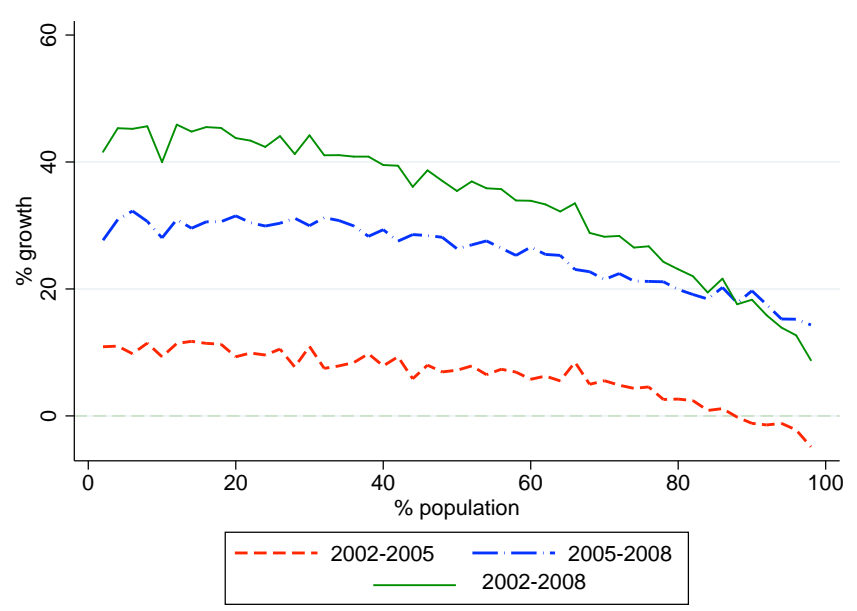

Figure 7: Brazilian OGICs 2002-2005-2008. Source: Authors' calculation from PNAD.

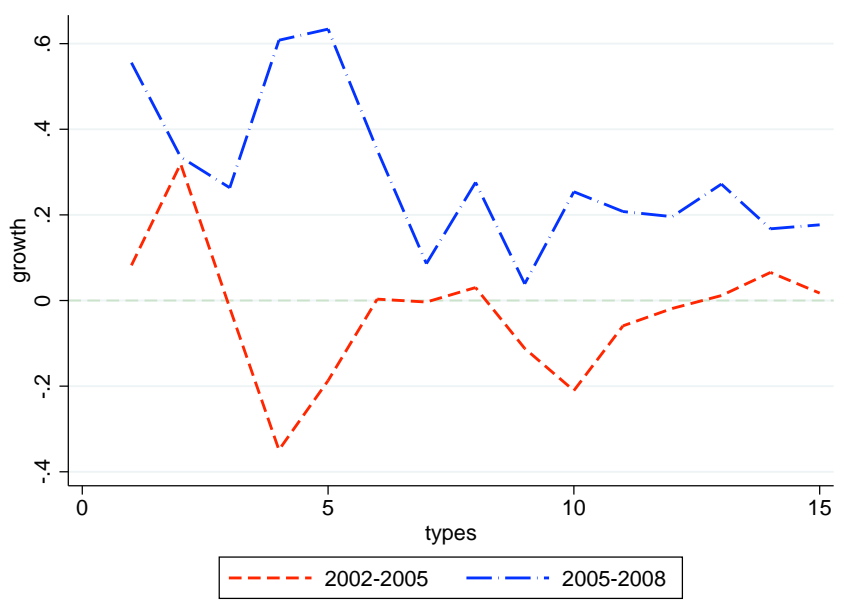


Figure 8: Brazilian OGICs 2002-2005-2008. Source: Authors' calculation from PNAD.

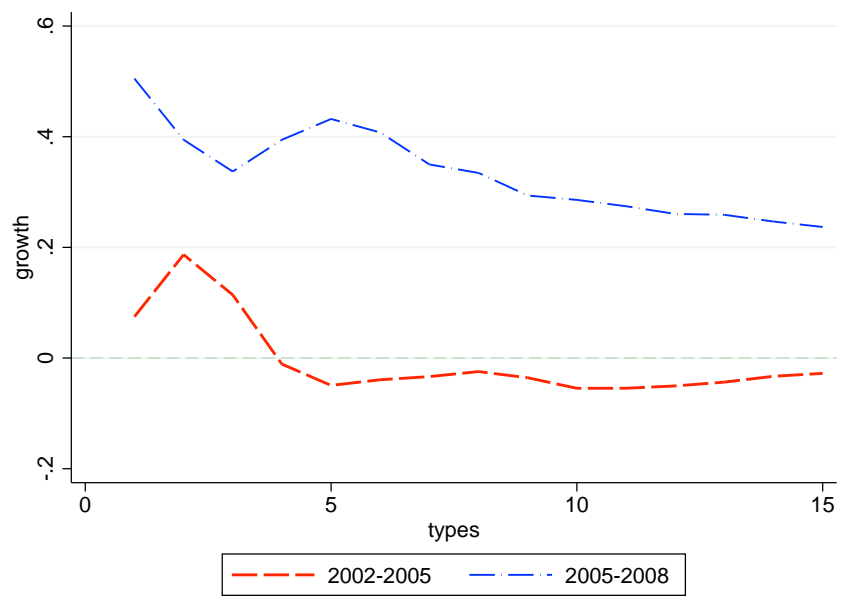

\section{Figures and Tables (Appendix)}

Figure 9: Italian GICs 1998-2002-2006. Source: Authors' calculation from SHIW.

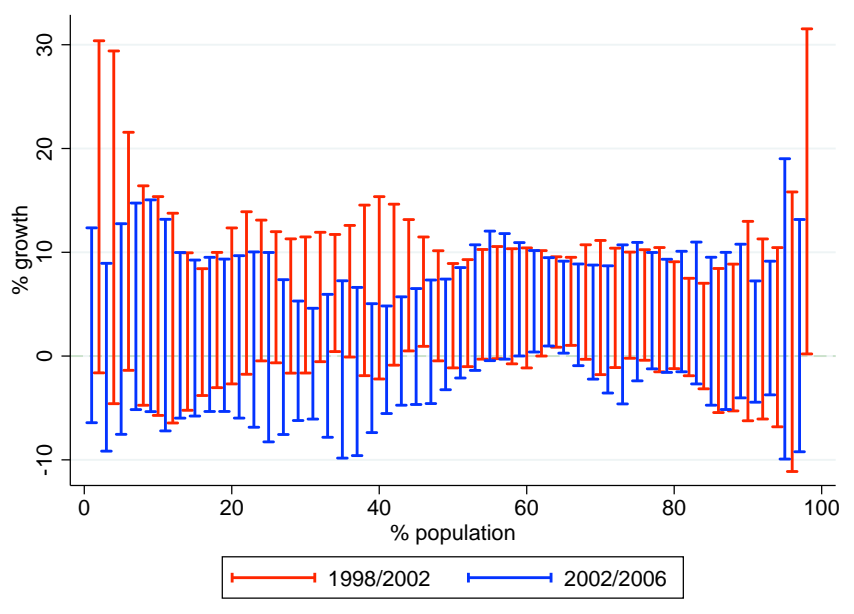


Table 2: 1998-2002 descriptive statistics (4-year panel). Source: Authors' calculation from SHIW

\begin{tabular}{llcccll}
\hline rank 98 & area & education & occupation & $q_{i}^{98}$ & $\mu_{i}^{98}$ & $\mu_{i}^{02}$ \\
\hline \hline 1 & South & Sec./tert. & Blue c./not in l.f. & 0.0101 & 6628.15 & 8161.74 \\
2 & South & No-edu/elem. & Blue c./not in l.f. & 0.1914 & 7814.47 & 7969.86 \\
3 & South & No-edu/elem. & White c. & 0.1097 & 8671.90 & 9112.20 \\
4 & South & No-edu/elem. & Executive & 0.0074 & 9552.87 & 9488.67 \\
5 & Centre & No-edu/elem. & White c. & 0.0481 & 9576.10 & 12163.99 \\
6 & South & Sec./tert. & White c. & 0.0332 & 9735.26 & 10634.27 \\
7 & Centre & No-edu/elem. & Blue c./not in l.f. & 0.0873 & 10139.11 & 11088.26 \\
8 & North & No-edu/elem. & Blue c./not in l.f. & 0.2028 & 11627.92 & 12125.37 \\
9 & South & Sec./tert. & Executive & 0.0250 & 11888.34 & 12648.81 \\
10 & North & Sec./tert. & White c. & 0.0645 & 13779.78 & 15198.73 \\
11 & North & No-edu/elem. & White c. & 0.1366 & 13877.98 & 12977.79 \\
12 & Centre & Sec./tert. & White c. & 0.0130 & 14424.79 & 14317.74 \\
13 & North & Sec./tert. & Blue c./not in l.f. & 0.0194 & 14839.41 & 16886.87 \\
14 & Centre & Sec./tert. & Blue c./not in l.f. & 0.0073 & 16063.54 & 14916.54 \\
15 & North & No-edu/elem. & Executive & 0.0082 & 16476.35 & 14109.81 \\
16 & Centre & Sec./tert. & Executive & 0.0093 & 17255.09 & 19073.36 \\
17 & Centre & No-edu/elem. & Executive & 0.0015 & 20350.81 & 19414.49 \\
18 & North & Sec./tert. & Executive & 0.0253 & 20633.05 & 24126.90 \\
\hline
\end{tabular}


Table 3: 2002-2006 descriptive statistics (4-year panel). Source: Authors' calculation from SHIW

\begin{tabular}{llcccll}
\hline rank 02 & area & education & occupation & $q_{i}^{02}$ & $\mu_{i}^{02}$ & $\mu_{i}^{06}$ \\
\hline \hline 1 & South & No-edu/elem. & Executive & 0.0046 & 7614.34 & 9994.33 \\
2 & South & No-edu/elem. & Blue c./not in l.f. & 0.1758 & 8343.45 & 8200.30 \\
3 & South & No-edu/elem. & White c. & 0.1280 & 9679.26 & 9722.65 \\
4 & Centre & No-edu/elem. & Blue c./not in l.f. & 0.0998 & 10809.23 & 11672.70 \\
5 & South & Sec./tert. & White c. & 0.0399 & 11181.27 & 10894.90 \\
6 & North & No-edu/elem. & Blue c./not in l.f. & 0.1874 & 11777.86 & 12443.61 \\
7 & Centre & Sec./tert. & Blue c./not in l.f. & 0.0114 & 11908.11 & 12456.78 \\
8 & South & Sec./tert. & Blue c./not in l.f. & 0.0157 & 12283.94 & 11153.89 \\
9 & Centre & No-edu/elem. & White c. & 0.0384 & 12846.91 & 12774.39 \\
10 & North & Sec./tert. & Blue c./not in l.f. & 0.0329 & 13241.98 & 15953.10 \\
11 & South & Sec./tert. & Executive & 0.0221 & 13453.16 & 13995.27 \\
12 & North & No-edu/elem. & Executive & 0.0120 & 13982.01 & 17208.36 \\
13 & North & No-edu/elem. & White c. & 0.1084 & 14233.62 & 13490.15 \\
14 & Centre & Sec./tert. & White c. & 0.0205 & 14275.85 & 15843.21 \\
15 & Centre & No-edu/elem. & Executive & 0.0023 & 15339.54 & 10261.34 \\
16 & Centre & Sec./tert. & Executive & 0.0121 & 17316.67 & 19052.49 \\
17 & North & Sec./tert. & White c. & 0.0469 & 17698.90 & 19240.36 \\
18 & North & Sec./tert. & Executive & 0.0420 & 22376.14 & 21743.89 \\
\hline
\end{tabular}


Table 4: 1998-2006 descriptive statistics (8-year panel). Source: Authors' calculation from $S H I W$.

\begin{tabular}{llccccc}
\hline rank 98 & area & education & occupation & $q_{i}^{98}$ & $\mu_{i}^{98}$ & $\mu_{i}^{06}$ \\
\hline \hline 1 & South & Sec./tert. & Blue c./not in l.f. & 0.0125 & 6555.00 & 7693.48 \\
2 & South & No-edu/elem. & Executive & 0.0063 & 7846.22 & 7631.37 \\
3 & South & No-edu/elem. & Blue c./not in l.f. & 0.1840 & 8440.52 & 8374.97 \\
4 & South & No-edu/elem. & White c. & 0.0945 & 8622.23 & 9944.43 \\
5 & Centre & No-edu/elem. & White c. & 0.0522 & 9329.79 & 12432.31 \\
6 & Centre & No-edu/elem. & Blue c./not in l.f. & 0.0910 & 9834.42 & 11087.19 \\
7 & Centre & Sec./tert. & White c. & 0.0106 & 10722.60 & 14627.56 \\
8 & North & No-edu/elem. & Blue c./not in l.f. & 0.2507 & 11210.97 & 11379.38 \\
9 & South & Sec./tert. & White c. & 0.0307 & 11341.53 & 10819.66 \\
10 & South & Sec./tert. & Executive & 0.0278 & 12048.21 & 12990.20 \\
11 & Centre & Sec./tert. & Blue c./not in l.f. & 0.0053 & 12256.08 & 10496.23 \\
12 & North & No-edu/elem. & White c. & 0.1105 & 12646.93 & 12195.16 \\
13 & North & Sec./tert. & Blue c./not in l.f. & 0.0194 & 13336.49 & 13173.95 \\
14 & North & Sec./tert. & White c. & 0.0642 & 13808.27 & 16289.64 \\
15 & Centre & Sec./tert. & Executive & 0.0099 & 16410.18 & 16605.04 \\
16 & North & No-edu/elem. & Executive & 0.0075 & 17480.88 & 15991.58 \\
17 & Centre & No-edu/elem. & Executive & 0.0018 & 19229.14 & 19593.49 \\
18 & North & Sec./tert. & Executive & 0.0212 & 21792.37 & 26941.93 \\
\hline
\end{tabular}

Figure 10: Italian OGICs 1998-2002-2006. Source: Authors' calculation from SHIW.

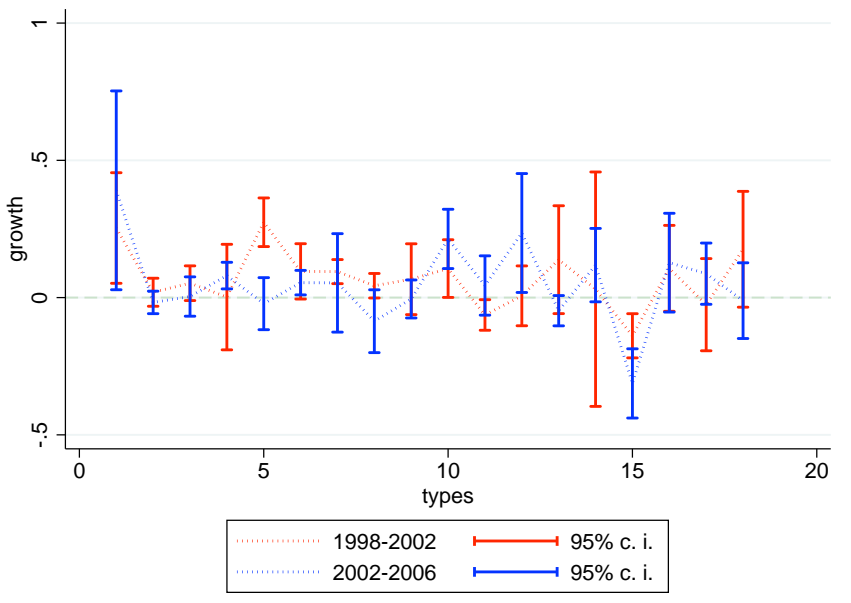


Figure 11: Proposition 1: OGICs 1998-2002-2006. Source: Authors' calculation from SHIW

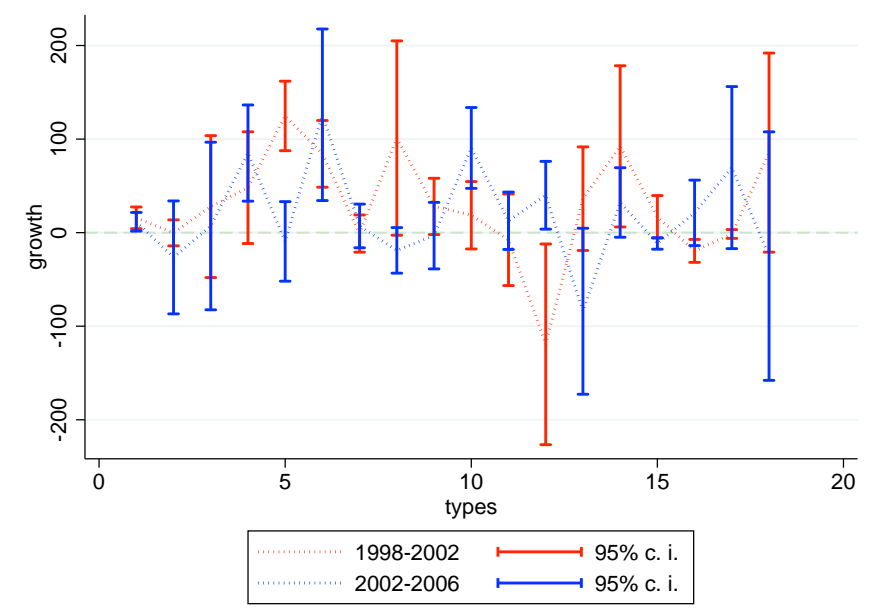

Figure 12: Proposition 2: OGICs 1998-2002-2006. Source: Authors' calculation from SHIW

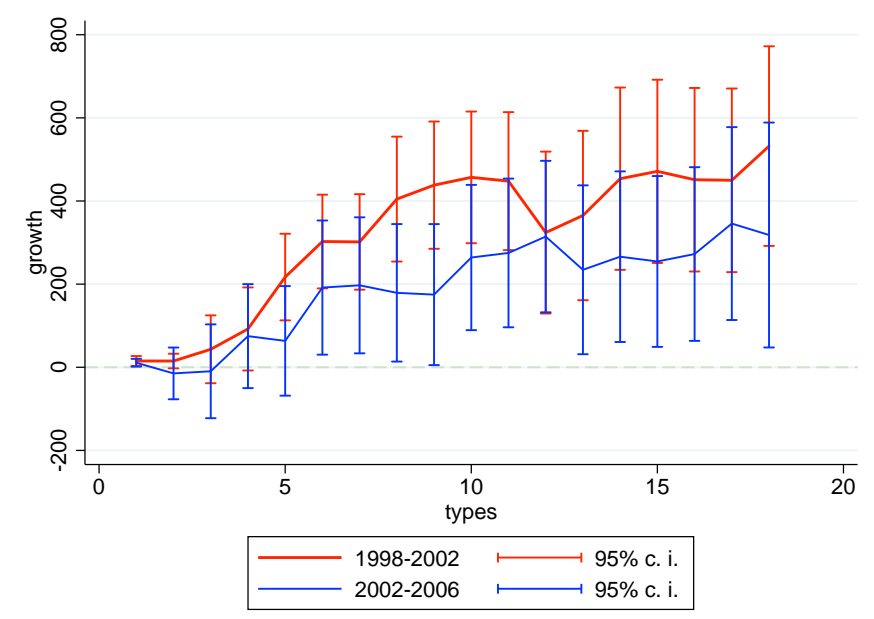


Figure 13: Brazilian GICs 2002-2005-2008. Source: Authors' calculation from PNAD.

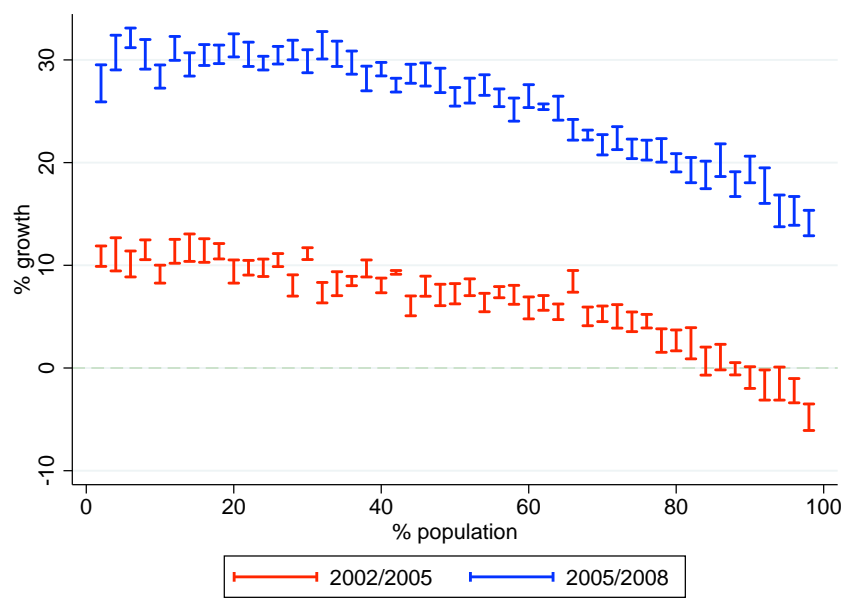

Table 5: 2002-2005 descriptive statistics. Source: Authors' calculation from PNAD

\begin{tabular}{cccccccc}
\hline rank 02 & rank 05 & race & region & $q_{i}^{02}$ & $q_{i}^{05}$ & $\mu_{i}^{02}$ & $\mu_{i}^{05}$ \\
\hline \hline 1 & 2 & black-mixed & Northeast & 0.2227 & 0.2229 & 276.48 & 298.67 \\
2 & 6 & indigenous & Northeast & 0.0007 & 0.0006 & 319.27 & 400.19 \\
3 & 3 & black-mixed & North & 0.0381 & 0.0541 & 325.53 & 318.37 \\
4 & 7 & black-mixed & South & 0.0270 & 0.0291 & 370.67 & 412.37 \\
5 & 8 & black-mixed & Center-west & 0.0300 & 0.0307 & 410.29 & 432.53 \\
6 & 9 & black-mixed & Southeast & 0.1448 & 0.1605 & 412.16 & 440.47 \\
7 & 4 & indigenous & North & 0.0002 & 0.0002 & 441.30 & 332.74 \\
8 & 10 & white-east asian & Northeast & 0.1093 & 0.1018 & 447.17 & 460.71 \\
9 & 1 & indigenous & Center-west & 0.0003 & 0.0002 & 464.02 & 235.02 \\
10 & 5 & indigenous & South & 0.0002 & 0.0003 & 484.86 & 355.29 \\
11 & 11 & white-east asian & North & 0.0146 & 0.0168 & 519.53 & 490.28 \\
12 & 12 & indigenous & Southeast & 0.0004 & 0.0004 & 595.04 & 602.99 \\
13 & 14 & white-east asian & South & 0.1310 & 0.1244 & 649.91 & 697.12 \\
14 & 13 & white-east asian & Center-west & 0.0245 & 0.0239 & 653.72 & 658.40 \\
15 & 15 & white-east asian & Southeast & 0.2561 & 0.2341 & 766.48 & 783.01 \\
\hline
\end{tabular}


Table 6: 2005-2008 descriptive statistics. Source: Authors' calculation from PNAD

\begin{tabular}{|c|c|c|c|c|c|c|c|}
\hline rank 05 & rank 08 & race & region & $q_{i}^{05}$ & $q_{i}^{08}$ & $\mu_{i}^{05}$ & $\mu_{i}^{08}$ \\
\hline 1 & 4 & indigenous & Center-west & 0.0002 & 0.0003 & 235.02 & 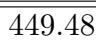 \\
\hline 2 & 1 & black-mixed & Northeast & 0.2229 & 0.2271 & 298.67 & 383.13 \\
\hline 3 & 3 & black-mixed & North & 0.0541 & 0.0556 & 318.37 & 403.50 \\
\hline 4 & 5 & indigenous & North & 0.0002 & 0.0003 & 332.74 & 467.03 \\
\hline 5 & 10 & indigenous & South & 0.0003 & 0.0004 & 355.29 & 575.12 \\
\hline 6 & 2 & indigenous & Northeast & 0.0006 & 0.0010 & 400.19 & 391.71 \\
\hline 7 & 6 & black-mixed & South & 0.0291 & 0.0319 & 412.37 & 526.75 \\
\hline 8 & 9 & black-mixed & Center-west & 0.0307 & 0.0321 & 432.53 & 557.04 \\
\hline 9 & 7 & black-mixed & Southeast & 0.1605 & 0.1633 & 440.47 & 538.04 \\
\hline 10 & 8 & white-east asian & Northeast & 0.1018 & 0.1018 & 460.71 & 549.13 \\
\hline 11 & 11 & white-east asian & North & 0.0168 & 0.0164 & 490.28 & 598.10 \\
\hline 12 & 12 & indigenous & Southeast & 0.0004 & 0.0005 & 602.99 & 672.61 \\
\hline 13 & 13 & white-east asian & Center-west & 0.0239 & 0.0237 & 658.40 & 809.25 \\
\hline 14 & 14 & white-east asian & South & 0.1244 & 0.1199 & 697.12 & 836.10 \\
\hline 15 & 15 & white-east asian & Southeast & 0.2341 & 0.2256 & 783.01 & 922.90 \\
\hline
\end{tabular}

Figure 14: Brazilian OGICs 2002-2005-2008. Source: Authors' calculation from PNAD.

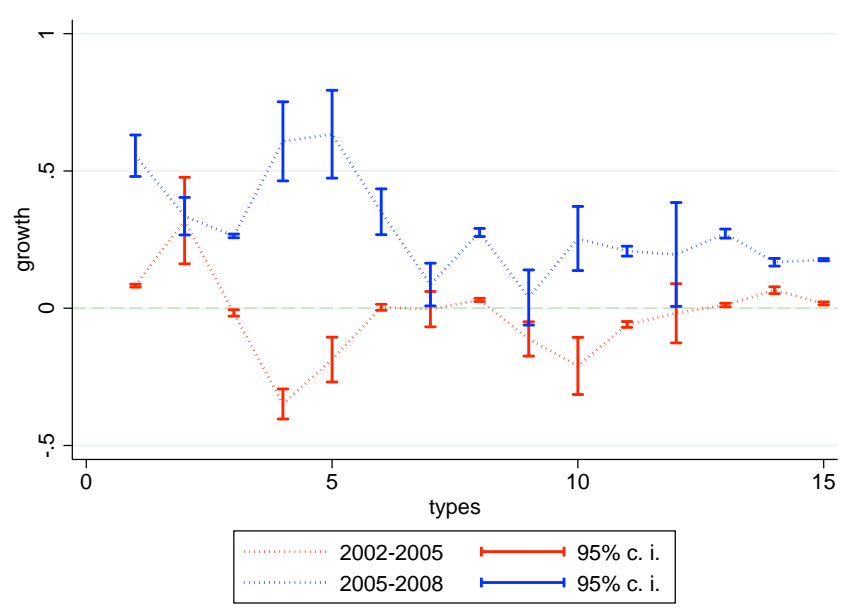


Figure 15: Brazilian OGICs 2002-2005-2008. Source: Authors' calculation from PNAD.

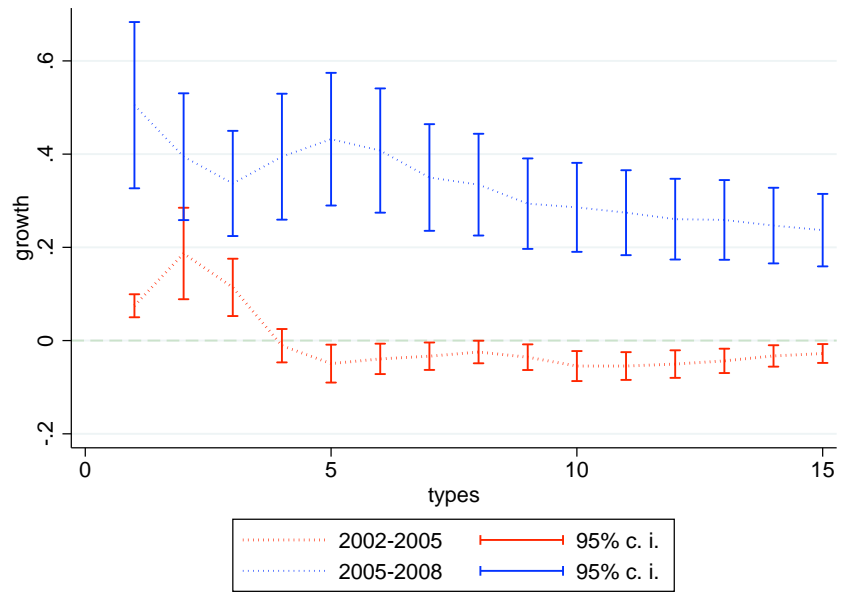

